\title{
Measuring rotation periods of solar-like stars using TIGRE
}

\section{A study of periodic Call $\mathrm{H}+\mathrm{K}$ S-index variability}

\author{
A. Hempelmann ${ }^{1}$, M. Mittag ${ }^{1}$, J. N. Gonzalez-Perez ${ }^{1}$, J. H. M. M. Schmitt ${ }^{1}$, K. P. Schröder ${ }^{2}$, and G. Rauw ${ }^{3}$ \\ 1 Universität Hamburg, Hamburger Sternwarte, Gojenbergsweg 112, 21029 Hamburg, Germany \\ e-mail: ahempelmann@hs.uni-hamburg.de \\ 2 Universidad de Guanajuato, Departamento de Astronomía, 37320 Guanajuato, Mexico \\ ${ }^{3}$ Institut d'Astrophysique et de Géophysique, Université de Liège, 4000 Liège, Belgium
}

Received 16 July 2015 / Accepted 20 November 2015

\begin{abstract}
Context. The rotation period of a star is a key parameter both for the stellar dynamo that generates magnetic fields as well as for stellar differential rotation.

Aims. We present the results from the first year of monitoring a sample of solar-like stars by the TIGRE facility in Guanajuato (Mexico), which will study rotation in solar analogs.

Methods. TIGRE is an automatically operating $1.2 \mathrm{~m}$ telescope equipped with an Échelle spectrograph with a spectral resolution of 20000 , which covers a spectral range of between 3800 and $8800 \AA$. A main task is the monitoring the stellar activity of cool stars, mainly in the emission cores of the CaII $\mathrm{H}$ and $\mathrm{K}$ lines. We observed a number of stars with a sampling between 1-3 days over one year.

Results. A total number of 95 stars were observed between August 12013 and July 31 2014, the total number of spectra taken for this program was appoximately 2700 . For almost a third of the sample stars the number of observations was rather low (less than 20), mainly because of bad weather. Fifty-four stars show a periodic signal but often with low significance. Only 24 stars exhibit a significant period. We interpret these signals as stellar rotation. For about half of them the rotation periods were already previously known, in which case our period measurements are usually in good agreement with the literature values. Besides the periodic signals, trends are frequently observed in the time series.

Conclusions. TIGRE is obviously able to detect stellar rotation periods in the $\mathrm{CaII} \mathrm{H}+\mathrm{K}$ emission cores when the time series contains a sufficient number of data points. However, this is frequently not achievable during the wet summer season in Guanajuato. Hence, future estimates of rotation periods will concentrate on stars that are observable during the winter season from October until April.
\end{abstract}

Key words. stars: activity - stars: solar-type - stars: rotation

\section{Introduction}

Stellar rotation and specifically the phenomenon of stellar differential rotation are thought to play a key role in the generation of stellar magnetic fields in cool stars with outer convection zones (Rüdiger 1989; Rüdiger \& Hollerbach 2004), since both the so-called $\alpha$-effect and the $\Omega$-effect depend on the rotation and differential rotation of the star. Therefore, these magnetic fields are thought to be the ultimate source for the plethora of "activity" phenomena on late-type stars, which can be summarized as the rotation-activity connection. Moreover, there seems to be a very direct link between the rotation period and the age of a star which opens a new and relatively straightforward method for determining stellar age (Meibom et al. 2015). Consequently, measurements of stellar rotation are clearly fundamental for stellar astropyhsics, since rotation is a key parameter for the age and activity of the underlying star.

Unfortunately, measuring stellar rotation is not at all straightforward. The usual way to measure the rotation period of a spotted star is by photometry. If star spots are inhomogeneously distributed across the surface of a star, the stellar flux is modulated with the rotation period. Using the high-precision, more or less uninterrupted photometry obtained with the Kepler satellite, McQuillan et al. (2014) measured and published rotation periods of more than 30000 stars. Unfortunately, period measurements of stars with low levels of activity become difficult even from space. Taking the Sun for example, an analysis of a long time series of the total solar irradiance did not yield an unambiguous and significant signature of solar rotation. Only during solar minimum conditions can the (known) solar rotation period be unambiguously detected in the solar irradiance time series (Hempelmann 2003) and, with ground based photometry alone, the rotational modulation of the solar flux is not detectable.

Another method to determine stellar rotation is the measurement of the chromospheric emission in the cores of certain spectral lines like $\mathrm{CaII} \mathrm{H}+\mathrm{K}$ doublet or the $\mathrm{CaII}$ infrared triplet. Eberhard \& Schwarzschild (1913) were the first to propose measuring the solar analog of activity in cool stars by observations of the line core emission in the stellar $\mathrm{CaII} \mathrm{H}+\mathrm{K}$ lines. Half a century later this idea was realized by O.C. Wilson and his colleagues in the framework of the famous Mt. Wilson project 
(Wilson 1978; Vaughan et al. 1978; Noyes et al. 1984). Baliunas et al. (1995) give a summary of this project and present longterm measurements of the so-called S-index (see below) for a sample of more than 100 stars of late spectral type.

The emission in the cores of the CaII $\mathrm{H}+\mathrm{K}$ lines is mainly caused by faculae (plage) in the chromosphere of the underlying star. The more the chromosphere is covered with plage regions, the stronger the emission is relative to the absorption by the photosphere. On the Sun, plage regions might be present on the surface, even when there are no visible spots and it is the plage regions that make the Sun - on average - brighter at solar maximum than at solar minimum, while spots lead to a temporary flux reduction. The Mt. Wilson S-index provides a measure of the strength of this plage emission. It is defined as the ratio of the flux measured in two approximately $1 \AA$ broadbands, both centered on the cores of the two absorption lines ( $\mathrm{H}$ and $\mathrm{K})$, and the flux measured in two $20 \AA$ broadbands, located to the left and right of the $\mathrm{H}$ and $\mathrm{K}$ lines in the pseudo-continuum(Vaughan et al. 1978). In this fashion, a very large number of stars has been catalogued with their $S$-index values over the years (Duncan et al. 1991; Henry et al. 1996; Gray et al. 2003; Wright et al. 2004; Gray et al. 2006; Isaacson \& Fischer 2010; Jenkins et al. 2011).

The advantage of these S-index measurements can be demonstrated in the case of the Sun. On the Sun the amount of plage varies substantially with its 11-year spot cycle, hence the disk-integrated solar $S$-Index also varies cyclically with this period (Donahue \& Keil 1995; Baliunas et al. 1995; Hempelmann \& Donahue 1997). This allows the solar cycle to be determined from observations of the Sun as a star and similar long-term variations over years and decades are observed on other cool stars (spectral classes F to M), among them stars with cyclic behaviour like for the Sun (Baliunas et al. 1995; Hall et al. 2007).

Frequently, the solar plage distribution is usually concentrated in "active" regions, but not necessarily around Sun spots. As with spots, this non-uniform distribution can cause variability of the $S$-index, as well as on the time-scale of solar rotation. Therefore, the solar rotation period can be in fact detected from S-index time series taken over several months, and furthermore, even the fingerprints of solar differential rotation can be derived from such time series if they cover at least one solar cycle (Donahue 1993; Hempelmann \& Donahue 1997). Determining the rotation periods from an S-index time series was successfully applied to stars, too; see, for example, Stimets \& Giles (1980), Vaughan et al. (1981), Noyes et al. (1984), Baliunas et al. (1985), and Wright et al. (2004). Similiarly, the rotation period was monitored over years and decades for a number of stars where, indeed, systematic variations in the stellar activity were found and interpreted as the result of stellar differential rotation (SRD), and active belt migration as analogs to the solar butterfly diagram (Baliunas et al. 1985; Donahue \& Keil 1995).

The Mt.Wilson project was terminated at the end of the twentieth century. Several follow-up projects have been started since then to monitor the CaII S-Index of cool stars, for example at Lowell Observatory (Hall et al. 2007) and at the Teide Observatory by the STELLA project (Granzer et al. 2010). A new project is Telescopio Internacional de Guanajuato Robótico Espectroscópico (TIGRE), which is operated at the La Luz Observatorio in Mexico. The robotic TIGRE facility (Schmitt et al. 2014) started its regular operations in August 2013. As a robotic observatory, it is ideally suited for monitoring projects and one of the science goals of TIGRE is a systematic study of stellar rotation and activity. After a brief description of TIGRE and the TIGRE data reduction, we will present the first results of a monitoring of $95 \mathrm{cool}$ stars over one year.

\section{Observations}

\subsection{The TIGRE facility}

TIGRE is a $1.2 \mathrm{~m} \mathrm{f} / 8$ Cassegrain-Nasmyth telescope equipped with an Échelle spectrograph located at La Luz Observatory at $2400 \mathrm{~m}$ altitude near Guanajuato (Mexico). The modernised spectrograph Heidelberg Extended Range Optical Spectrograph (HEROS) covers the wavelength ranges 380-570 and $583-880 \mathrm{~nm}$ with a spectral resolution of $\lambda / \Delta \lambda \approx 20000$. The important feature of TIGRE is its fully automatic operation. Before starting an observational season, a list of target stars with parameters necessary for automatic operations is stored on the central TIGRE computer. When the central control software (CCS) requests a target to be observed, the "scheduler", which contains the "intelligent" part of the software, calculates a priority for each target of the list and, at the end, chooses the target of highest priority. Parameters for priority calculations include, among others, the visibility of the target during the night and at the beginning and end of exposure. A second important parameter is the history of observations of the target together with its required frequency of observations of this star. The CCS manages the complete system, including telescope, spectrograph, dome and auxiliary systems like the weather station. In case of non-optimal weather conditions like cirrostratus clouds or very bad seeing, the nominal exposure time of the target is increased to obtain the required $\mathrm{S} / \mathrm{N}$ in the spectrum. A detailed description of TIGRE is given by Schmitt et al. (2014).

For all practical purposes TIGRE operates with (usually) no direct human intervention. In 2014, a total of about 5000 spectra were taken. TIGRE is located at the northern edge of the tropics, where there is a rainy season during summer time with shorter nights, while the dry season is during the winter time with longer nights. Therefore, summer targets are more difficult to observe with the same frequency as winter targets, and one question to be answered during the first one-year season of TIGRE operations was, therefore, whether the summer season is suitable for monitoring tasks with dense sampling, as is required for measuring rotation periods.

\subsection{Data reduction}

The TIGRE data-reduction pipeline, including an automated wavelength calibration, is also fully automatic. It is implemented in the IDL environment and uses the data-reduction package REDUCE (Piskunov \& Valenti 2002), with some special adaption for the HEROS context. The reduction process includes all standard and necessary reduction steps for Échelle spectra.

The level of dark current in the ANDOR CCD cameras used to record the spectra is very low, implying that the dark noise lies well below the read-out noise and hence, the mean dark level is removed during background correction. For flat fielding we use a tungsten lamp spectrum, and finally, from standard star spectra we compute a correction function, which is then used to remove any remaining residuals from the blaze correction of the spectra.

The spectra of a ThAr hallow cathode lamp are used for the wavelength calibration. These calibration spectra are taken at the beginning and the end of a night. The extracted ThAr spectra are cross-correlated with a master ThAr spectrum as a reference to determine the mean wavelength shift. A detailed description of the data-reduction pipeline is given by Mittag et al. (2010). 


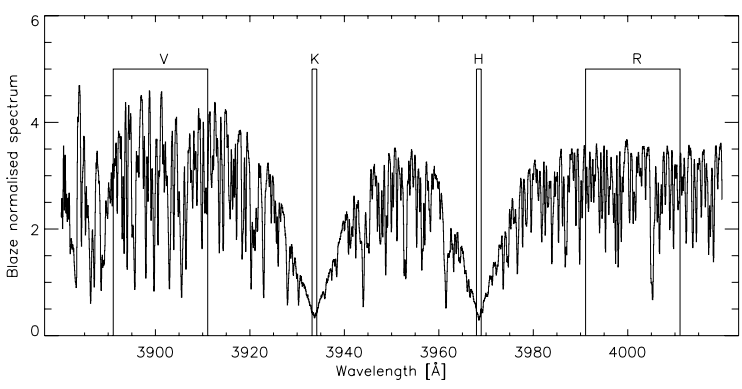

Fig. 1. Solar (Moon) CaII H+K spectrum with the TIGRE band passes.

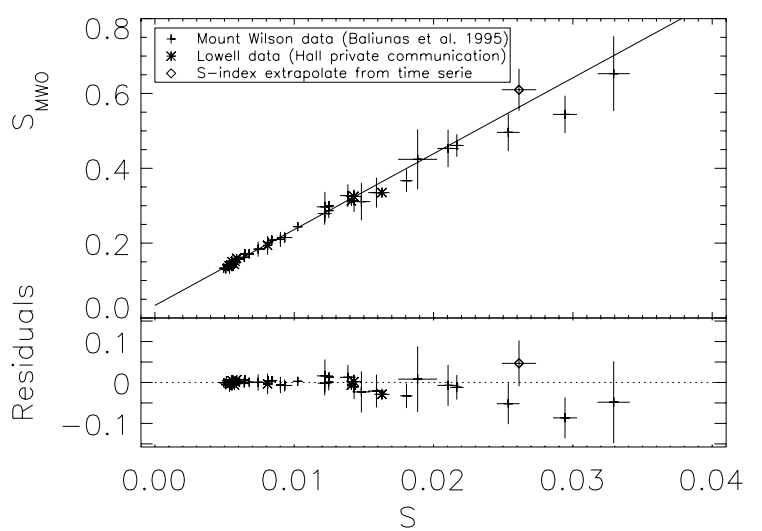

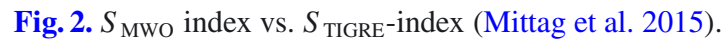

\subsection{S-indexes}

The Mt.Wilson $S$-index (hereafter $S_{\text {Mwo }}$ index) is the most commonly used activity index in the optical spectral range. Traditionally, it is defined as the ratio of the counts in the $\mathrm{CaII} \mathrm{H}+\mathrm{K}$ line centers in triangular bandpasses with a FWHM of $1.09 \AA$ for MS stars over two $20 \AA$ pseudo-continuum bandpasses that are centered at $3901.07 \AA$ and $4001.07 \AA$ (Vaughan et al. 1978; Duncan et al. 1991). The TIGRE $S$-index (hereafter $S_{\text {TIGRE-index) is calculated (actually in the data reduction }}$ pipeline) from the blaze-normalised and Échelle order merged spectra. Here a rectangular $1.00 \AA$ bandpass is used to measure the flux in the CaII $\mathrm{H}+\mathrm{K}$ cores, while the side bandpasses are the same as for $S_{\text {MWO }}$ (Mittag et al. 2015, see also Fig. 1). Clearly, it is very important to determine the relationship between $S_{\text {TIGRE }}$ and $S_{\text {MWO }}$ to make our TIGRE measurements comparable with those of the Mt. Wilson project and the vast body of available $\mathrm{S}$-index measurements. For this purpose, a set of 45 calibration stars was selected (Mittag et al. 2015), for which well-

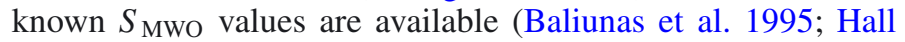
et al. 2007). These stars are also known to be relatively constant in their activity levels. The result is shown in Fig. 2, a linear least-squares fit yields the following conversion relationship:

$S_{\text {MWO }}=(0.033 \pm 0.003)+(20.3 \pm 0.4) \cdot S_{\mathrm{TIGRE}}$.

In the following, all TIGRE measurements are transformed into the $S_{\text {MWO }}$ index using Eq. (1). The exposure times of the spectra were chosen so that a typical uncertainty of $1 \%$ of $S_{\text {TIGRE }}$ is reached, including a possible correction because of bad seeing or high extinction.

\section{Results}

\subsection{Sample characteristics}

So far, we have selected a sample of 95 stars from the solar neighborhood (within $\approx 20-30$ pc) mostly on or near the main sequence. Many of these stars have $B-V$ values around the solar one of $B-V=0.65$, and are henceforward referred to as "sisters of the Sun". Additionally, our sample includes further stars which were observed with other scientific objectives and where the determination of the rotation period was only a by-product. Our ultimate goal is a catalog of rotation periods of a volume-limited sample of sisters of the Sun, visible from Guanajuato. Our mixed sample, presented in this paper, is only a first step in this direction to demonstrate the feasibility of the chosen approach, to check which time series result in robust period estimates, and to compare our results to literature values. Our sample stars and our monitoring results are listed in Table A.1.

The actual list of stars to be observed with TIGRE is always much longer than our list of stars, since other science targets are also observed. With a few exceptions, all stars have the same scientific priority. Hence, the automatic scheduling process chooses the stars to be observed mainly according to the conditions of visibility and, according to the history of observations. For example, when a star is to be observed every three days, its observational priority will be low one day after the last observation, moderate after two days, but high after three days and more. In this case, the actual observational statistics will depend on the weather conditions and also on the total number of stars competing with each other. It is obvious from Table A.1 that the number of observations for the stars that are mainly visible during the wet season (May until September) is significantly lower than for stars in the winter season.

\subsection{Data analysis and period determinations}

The majority of our sample stars shows a long-tem trend in the recorded time series. If this trend continues to go in the same direction, this would be a strong indication of instrumental problems. However, this is not the case: the number of stars with increasing trends (30) balances with the stars of decreasing trends (28), while 37 stars show no trend.

Trends must be eliminated before performing a period analysis; we subtracted a polynomial fit of the time series from the data and carried out a Lomb-Scargle analysis on the de-trended data (Horne \& Baliunas 1986); all of our results are listed in Table A.1, Cols. 6-8. Significance $(\sigma)$ levels 1-3 mean: 68-95\% significance for $\sigma=1,95-99.6$ for $\sigma=2$, and $\geq 99.7$ for $\sigma=3$. The significance calculation is based on Eq. (2) in the paper by Baliunas et al. (1995), the $\Delta \mathrm{P}$ calculation is according to Kovacs (1981). We consider the cases of $\sigma=2-3$ as confident detections, while the $\sigma=1$ cases are more like suggested signals than really confident detections. In Fig. 3 we show typical cases in our monitoring samples that yielding either a non-detection or signal detections of the different levels of significance and with different long-term trends. In the case of HD 45067 (upper left in Fig. 3) we find neither a trend nor a period, in the case of HD 39881 (upper right in Fig. 3) a linear downward trend and a period at the $1 \sigma$ level; in the case of HD 72905 (bottom left in Fig. 3) there is again no trend and a period at the $2 \sigma$ level, which agrees with the literature to within five percent; and finally HD 37395 (bottom right in Fig. 3), which appears to show a quadratic trend and a period above the $3 \sigma$ level, which agrees with the literature value to within a few percent.

In spite of their sparse sampling, there are five stars from the summer season with signal detections $(2 \sigma)$ or suggested detections $(1 \sigma)$ : HD 168009, HD 201091, HD 207978, HD 217014 , and HD 224930. Thus, even during the summer season it is possible to detect a rotational signal from a poorly-sampled time 

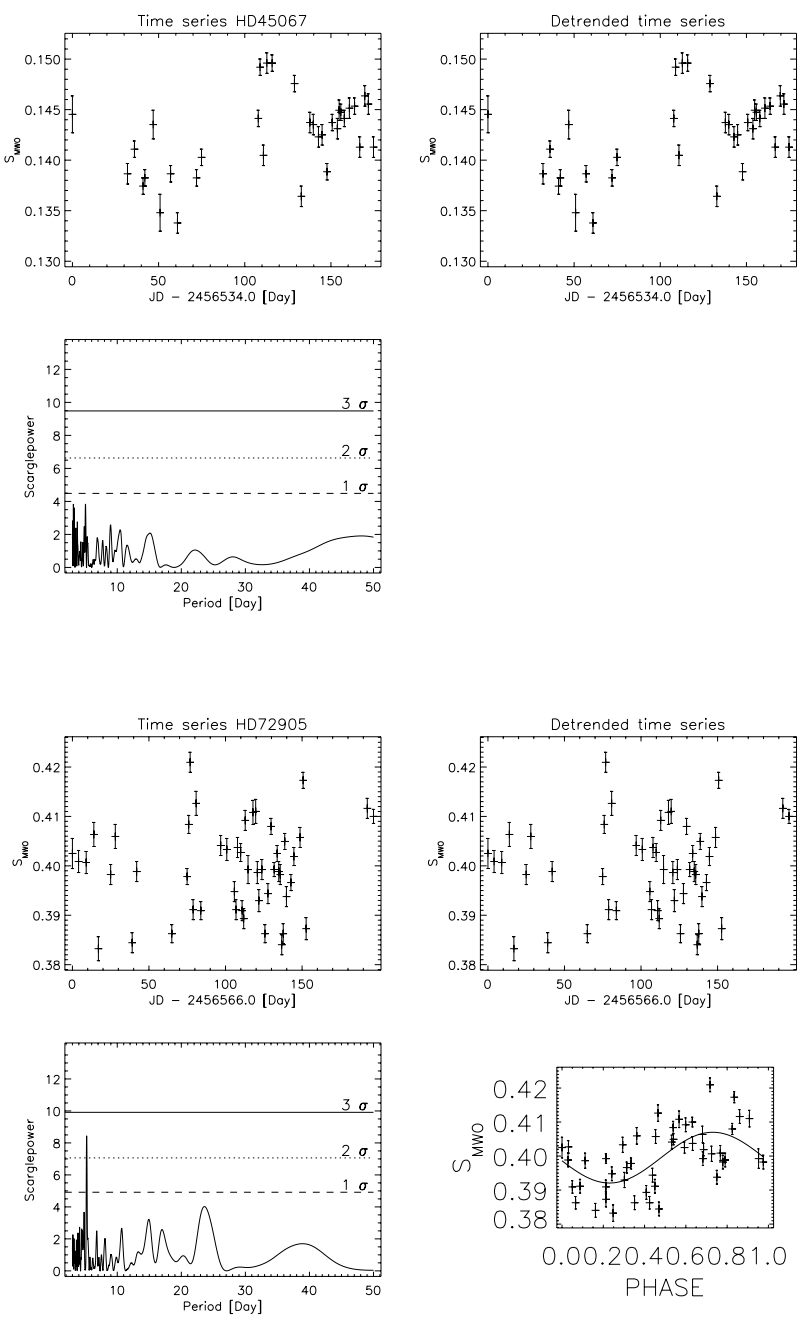
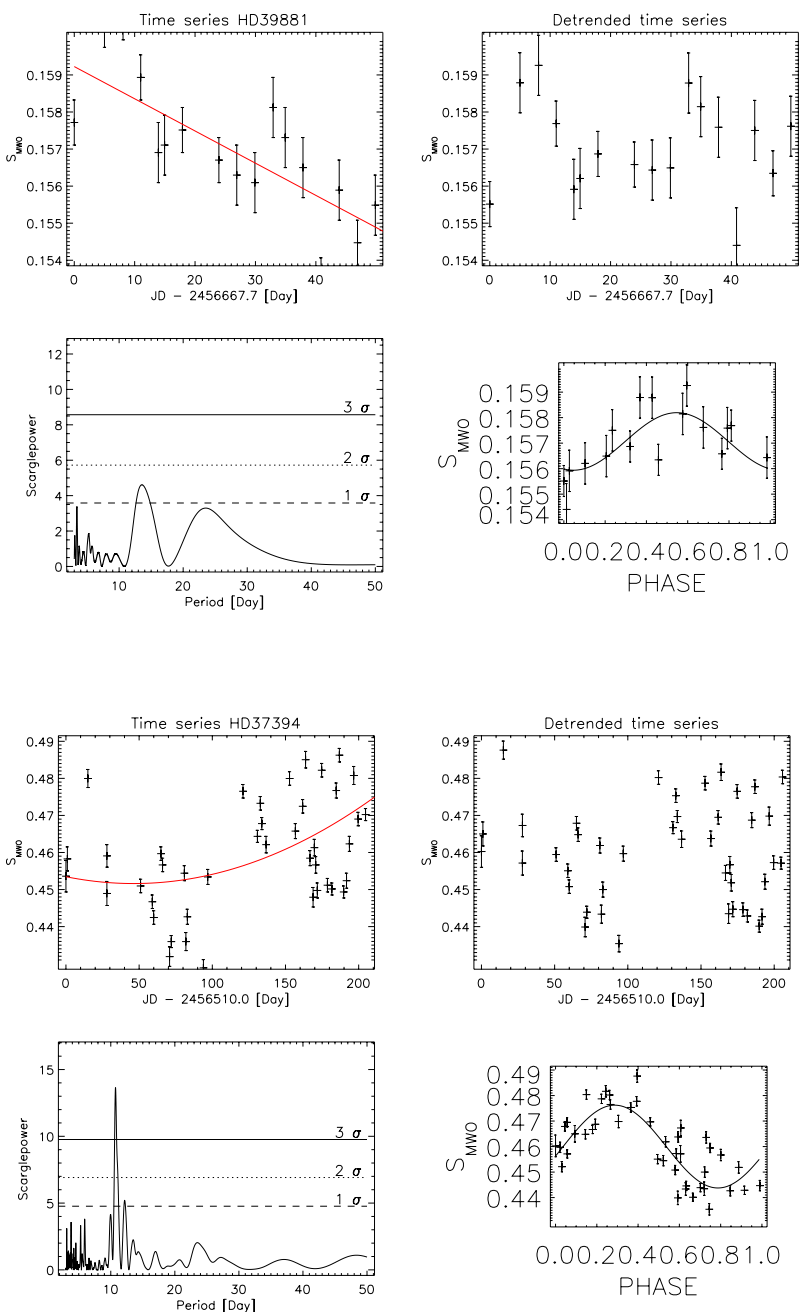

Fig. 3. Four examples of qualitatively different signal detections. Top left: no signal (HD 45067). Top right: $1 \sigma$ signal (HD 39881). Bottom left: $2 \sigma$ detection (HD 72905). Bottom right: $3 \sigma$ detection (HD 37394). Each figure contains the original time series (top left), including a polynomial fit (full line) to eliminate a long-term trend, the time series corrected for a long-term trend (top right), the Scargle periodogram (bottom left) and a phase plot (bottom right).

series, given a relatively big amplitude of the $S$-index oscillation. At least two stars (HD 20530 and HD 37124) have excellent visibility during the winter season but with very few observations. Obviously, the automatic scheduling process failed to produce adequate observations for these stars, the reason for which could not be identified.

\section{3. "Old" and "new" periods}

To assess the confidence of our findings, we made a comparison with earlier findings obtained by other authors and other instruments. In Table A.1, Cols. 9-10 the periods with references taken from the literature are listed. These values versus our findings are depicted in Fig. 4, which shows that the vast majority of our detections are in congruence with the earlier findings, even in the case of the formally non-significant $1 \sigma$ periods. Slight deviations can, in principle, be explained with surface differential rotation (SDR). SDR of the Sun, in connection with the solar butterfly diagram, which leads to a variation range of the rotation period of $10 \%$ (see also Hempelmann \& Donahue 1997). However, stellar investigations of SDR have yielded this variation range as being quite typical although the scatter in the SDR

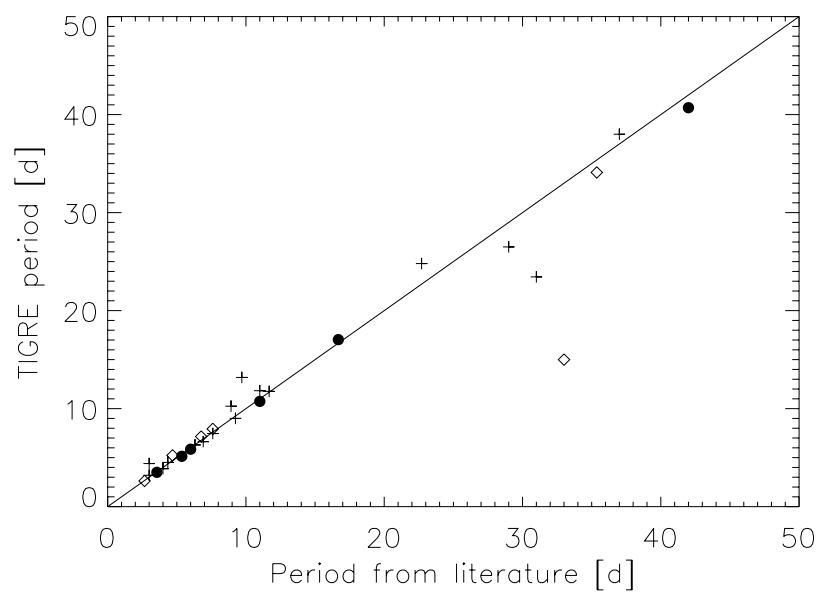

Fig. 4. Periods from TIGRE observations vs. values from literature. Full circles: $3 \sigma$ TIGRE detections; diamonds: $2 \sigma$; crosses: $1 \sigma$.

range is up to $30 \%$ and, even more in a few cases (Donahue et al. 1996). Bearing this in mind, the scatter around the zero deviation line in Fig. 4 may be mainly explained by SDR. 
The case of HD 13421 is quite curious. Here the strongest signal at the $2 \sigma$ level is at 2.173 days, which is half the known rotation period of 4.35 days. A second signal of the $1 \sigma$ level with 4.51 days is in the SDR range of 4.35 days. An explanation for the $2.17 \mathrm{~d}$ period as a first harmonic of the rotation period seems physically unlikely. A possible alternative is that plage is concentrated in two regions on opposite longitudes, which leads to maximum power at half of the rotation period in the periodogram. The star is depicted with $4.51 \mathrm{~d}$ in Fig. 4. A similar situation is found for HD 224930, where we find almost half of the literature value.

There are several stars with a published rotation period but without a TIGRE detection, although the number of observations was sufficiently large. We interpret this as suggesting that an inhomogeneous distribution of plage on the stellar surface may be a temporary occasion or, plage may be absent during the minimum phase of a stellar activity cycle. The latter was the case several times during solar minimum between cycles 23 and 24 .

\section{Summary and conclusions}

We have monitored a sample of almost 100 solar-like stars over one year with the robotic TIGRE facility, located at the La Luz Observatory in central Mexico. The temporal density of the TIGRE sampling was determined by i) the human input requirements, such as "Take a single exposure every night"; ii) the weather conditions changing over the year and; iii) the competition with other TIGRE targets. In general, the weather conditions during the wet summer season led to a lower number of observations, in comparison to the winter season. This low number has certainly enhanced the threshold for signal detection and also has lowered the significance of the detected periods, yet our periods, even those determined at low significance, agree well with earlier detections by other facilities. In addition, we still need to work on the performance of the automatic scheduler to improve the temporal sampling. We are thus led to the conclusion that rotation periods can also be found with TIGRE for summer season stars, but we need to continue for one or more consecutive years to verify our results.

The majority of stars show trends in their time series that imply that other sources of variability other than the stellar rotation must exist. Because of the shortness of the time series, it is difficult to decide whether these fluctuations are part of the longterm stellar activity cycle or come from active region growth and decay. The latter could be the case for HD 23249 with a 158-day signal, HD 120136 with a 114-day period, and maybe also for HD 26923 with a 60-day period, in the latter two cases over two consecutive oscillations. In Table A.1 these three periods have a question mark. By continuing to monitor this over the next years and decades may answer this question.

From the 95 stars monitored in 2014, 54 showed a periodic signal at least above the $1 \sigma$ level: 29 stars showed a signal at the $1-2 \sigma$ level, 14 stars had signals at the $2-3 \sigma$ level and 10 stars showed $>3 \sigma$ detections. For 26 stars the rotation periods are new as far as we are aware, while for 28 stars we confirm earlier measurements. We note in particular that, in many cases, our detections at the $1-2 \sigma$ level match well with literature rotation values, but we also note that the rotation periods of 15 stars with literature values could not yet be confirmed with TIGRE. The relatively high number of stars with low significance signals led to the conclusion that the density of sampling shall be enhanced in the future to increase the number of data points for the time series. Furthermore, we are in the process of studying other chromospheric activity indicators such as the CaII infrared triplett and $\mathrm{H}_{\alpha}$, which are also covered by our TIGRE spectra, to check for rotational modulation and how well it correlates with the signals in the $\mathrm{S}$-index.

Acknowledgements. We acknowledge the continued support by various partners who helped to realize TIGRE. Firstly, the University of Hamburg, which gave support in terms of funding, manpower, and workshop resources. Furthermore, grants from the Deutsche Forschungsgemeinschaft (DFG) in various funding lines is gratefully acknowledged, as well as travel money from both the DFG, $\mathrm{DAAD}$, and Conacyt in several bilateral grants. The Liège contribution to the TIGRE is funded through an opportunity grant from the University of Liège. The Universities of Guanajuato and Liège and the Mexican state of Guanajuato shared the funding of the infrastructure required by TIGRE at the La Luz site, and there is continued support by the University of Guanajuato in terms of manpower and running costs of the facilities. We thank the (anonymous) referee for very helpful comments and suggestions. We thank Dr. U. Wolter for proof-reading.

\section{References}

Baliunas, S., Sokoloff, D., \& Soon, W. 1996, ApJ, 457, L99

Baliunas, S. L., Horne, J. H., Porter, A., et al. 1985, ApJ, 294, 310

Baliunas, S. L., Donahue, R. A., Soon, W. H., et al. 1995, ApJ, 438, 269

Baliunas, S. L., Donahue, R. A., Soon, W., \& Henry, G. W. 1998, in Cool Stars, Stellar Systems, and the Sun, eds. R. A. Donahue, \& J. A. Bookbinder, ASP Conf. Ser., 154, 153

Cincunegui, C., Diaz, R. F., \& Mauas, P. J. D. 2007, VizieR Online Data Catalog: $\mathrm{J} / \mathrm{A}+\mathrm{A} / 469 / 309$

Donahue, R. A. 1993, Ph.D. Thesis, New Mexico State University, University Park

Donahue, R. A., \& Keil, S. L. 1995, Sol. Phys., 159, 53

Donahue, R. A., Saar, S. H., \& Baliunas, S. L. 1996, ApJ, 466, 384

Duncan, D. K., Vaughan, A. H., Wilson, O. C., et al. 1991, ApJS, 76, 383

Eberhard, G., \& Schwarzschild, K. 1913, ApJ, 38, 292

Granzer, T., Weber, M., \& Strassmeier, K. G. 2010, Adv. Astron., 2010, 79

Gray, R. O., Corbally, C. J., Garrison, R. F., McFadden, M. T., \& Robinson, P. E. 2003, AJ, 126, 2048

Gray, R. O., Corbally, C. J., Garrison, R. F., et al. 2006, AJ, 132, 161

Hall, J. C., Lockwood, G. W., \& Skiff, B. A. 2007, AJ, 133, 862

Hempelmann, A. 2003, A\&A, 399, 717

Hempelmann, A., \& Donahue, R. A. 1997, A\&A, 322, 835

Henry, T. J., Soderblom, D. R., Donahue, R. A., \& Baliunas, S. L. 1996, AJ, 111, 439

Henry, G. W., Baliunas, S. L., Donahue, R. A., Fekel, F. C., \& Soon, W. 2000 , ApJ, 531, 415

Horne, J. H., \& Baliunas, S. L. 1986, ApJ, 302, 757

Isaacson, H., \& Fischer, D. 2010, ApJ, 725, 875

Jenkins, J. S., Murgas, F., Rojo, P., et al. 2011, A\&A, 531, A8

Kovacs, G. 1981, Ap\&SS, 78, 175

McQuillan, A., Mazeh, T., \& Aigrain, S. 2014, ApJS, 211, 24

Meibom, S., Barnes, S. A., Platais, I., et al. 2015, Nature, 517, 589

Mittag, M., Hempelmann, A., González-Pérez, J. N., \& Schmitt, J. H. M. M. 2010, Adv. Astron., 2010, 6

Mittag, M., Hempelmann, A., Gonzalez-Perez, J. N., \& Schmitt, J. H. M. M. 2015, in Cambridge Workshop on Cool Stars, Stellar Systems, and the Sun, eds. G. T. van Belle, \& H. C. Harris, 18, 549

Noyes, R. W., Hartmann, L. W., Baliunas, S. L., Duncan, D. K., \& Vaughan, A. H. 1984, ApJ, 279, 763

Piskunov, N. E., \& Valenti, J. A. 2002, A\&A, 385, 1095

Rüdiger, G. 1989, Differential rotation and stellar convection. Sun and the solar stars (Berlin: Akademic Verlag)

Rüdiger, G., \& Hollerbach, R. 2004, in The magnetic universe: geophysical and astrophysical dynamo theory (Wiley-VCH), 343

Schmitt, J. H. M. M., Schröder, K.-P., Rauw, G., et al. 2014, Astron. Nachr., 335, 787

Simon, T., \& Fekel, Jr., F. C. 1987, ApJ, 316, 434

Simpson, E. K., Baliunas, S. L., Henry, G. W., \& Watson, C. A. 2010, MNRAS, 408, 1666

Stimets, R. W., \& Giles, R. H. 1980, ApJ, 242, L37

Vaughan, A. H., Preston, G. W., \& Wilson, O. C. 1978, PASP, 90, 267

Vaughan, A. H., Preston, G. W., Baliunas, S. L., et al. 1981, ApJ, 250, 276

Wilson, O. C. 1978, ApJ, 226, 379

Wright, J. T., Marcy, G. W., Butler, R. P., \& Vogt, S. S. 2004, ApJS, 152, 261

Wright, N. J., Drake, J. J., Mamajek, E. E., \& Henry, G. W. 2011, ApJ, 743, 48 


\section{Appendix A: Data table}

In Table A.1 we present our results, together with other information on our sample stars. The first three columns contain the Henry Draper catalogue number together with brightness and color information, the fourth column gives the total number of observations. The 5th column indicates long-term trends in the time series: linear increase $(+)$, decrease $(-)$, nonlinear increase $(-/+)$ and nonlinear decrease (+/-). The 6-7th columns indicate a period extracted from Fourier analyses according to Horne \& Baliunas (1986), together with its one sigma uncertainty $\Delta \mathrm{P}$ (the latter was calculated according to Kovacs 1981). Column 8 describes the level of significance of the period detection (for an explanation see the caption in Table A.1). The rotation period,which is taken from the literature, is given in Col. 9, together with its reference in Col. 10. Column 11 contains the S-index averaged over the time series and transformed according to Eq. (1) into the Mt. Wilson value, while the last column gives the $1 \sigma$ variation of that value.

Table A.1. Characteristics of the sample stars.

\begin{tabular}{|c|c|c|c|c|c|c|c|c|c|c|c|}
\hline Star & V & $B-V$ & $N$ & Trend & Period [d] & $\Delta \mathrm{P}[\mathrm{d}]$ & Sig & $P_{\text {lit }}$ & Ref. & $\left\langle S_{\mathrm{MWO}}\right\rangle$ & $\sigma$ \\
\hline HD 739 & 5.25 & 0.40 & 15 & - & & & & & & 0.142 & 0.004 \\
\hline HD 1461 & 6.46 & 0.68 & 13 & + & & & & & & 0.156 & 0.003 \\
\hline HD 5133 & 7.17 & 0.94 & 12 & & & & & & & 0.430 & 0.013 \\
\hline HD 6582 & 5.17 & 0.69 & 20 & $+/-$ & & & & & & 0.175 & 0.005 \\
\hline HD 6920 & 5.68 & 0.56 & 25 & $+1-$ & & & & 13.1 & N84 & 0.198 & 0.006 \\
\hline HD 9826 & 4.10 & 0.54 & 30 & $-1+$ & & & & 14.0 & H0O & 0.152 & 0.004 \\
\hline HD 10307 & 4.96 & 0.62 & 22 & $-1+$ & & & & & & 0.152 & 0.005 \\
\hline HD 10476 & 5.24 & 0.84 & 20 & + & & & & 35.2 & D93 & 0.198 & 0.010 \\
\hline HD 10700 & 3.50 & 0.72 & 17 & $-/+$ & & & & 34 & B96 & 0.171 & 0.004 \\
\hline HD 13421 & 5.64 & 0.52 & 20 & + & $2.173,4.513$ & $0.002,0.09$ & 2,1 & 4.35 & S80 & 0.137 & 0.004 \\
\hline HD 16673 & 5.80 & 0.47 & 22 & $-1+$ & & & & 7.4 & W11 & 0.222 & 0.011 \\
\hline HD 17206 & 4.46 & 0.48 & 19 & $-1+$ & 3.847 & 0.009 & 1 & & & 0.223 & 0.009 \\
\hline HD 17925 & 6.05 & 0.86 & 33 & $-1+$ & 7.150 & 0.027 & 2 & 6.76 & W11 & $0.701 *$ & 0.023 \\
\hline HD 18940 & 7.06 & 0.62 & 22 & + & 10.25 & 0.08 & 1 & 8.92 & W11 & 0.295 & 0.016 \\
\hline HD 19019 & 6.76 & 0.52 & 19 & - & 13.18 & 0.12 & 1 & 9.70 & W11 & 0.216 & 0.006 \\
\hline HD 19373 & 4.05 & 0.59 & 35 & & & & & & & 0.146 & 0.001 \\
\hline HD 20619 & 7.04 & 0.34 & 26 & $+/-$ & 21.18 & 0.61 & 1 & & & 0.203 & 0.008 \\
\hline HD 20630 & 4.85 & 0.67 & 9 & & 9.0 & 0.2 & 1 & 9.24 & D96 & 0.380 & 0.014 \\
\hline HD 22049 & 3.73 & 0.88 & 23 & $-1+$ & 11.76 & 0.08 & 1 & 11.68 & W11 & $0.548^{*}$ & 0.016 \\
\hline HD 22072 & 6.17 & 0.89 & 28 & & & & & & & 0.142 & 0.003 \\
\hline HD 23249 & 3.54 & 0.92 & 32 & & $158 ?$ & 13 & 2 & & & 0.146 & 0.004 \\
\hline HD 25457 & 5.38 & 0.50 & 26 & & & & & 3.13 & W11 & 0.329 & 0.004 \\
\hline HD 25680 & 5.90 & 0.62 & 17 & - & & & & 9.1 & SF87 & 0.317 & 0.005 \\
\hline HD 25998 & 5.50 & 0.47 & 60 & & & & & 2.6 & N84 & 0.287 & 0.006 \\
\hline HD 26923 & 6.30 & 0.56 & 50 & $-1+$ & $60.4 ?$ & 2.1 & 2 & & & 0.286 & 0.007 \\
\hline HD 30495 & 5.50 & 0.64 & 35 & + & 11.83 & 0.09 & 1 & 11 & B96 & 0.281 & 0.012 \\
\hline HD 35296 & 5.00 & 0.52 & 56 & $+/-$ & 3.503 & 0.006 & 3 & 3.56 & W11 & 0.319 & 0.007 \\
\hline HD 37124 & 7.68 & 0.67 & 2 & & & & & & & 0.187 & 0.005 \\
\hline HD 37394 & 6.23 & 0.84 & 43 & $-/+$ & 10.74 & 0.03 & 3 & 11.0 & W11 & 0.460 & 0.015 \\
\hline HD 38858 & 5.97 & 0.64 & 16 & - & & & & & & 0.176 & 0.004 \\
\hline HD 39587 & 4.40 & 0.60 & 41 & $-1+$ & 5.136 & 0.009 & 3 & 5.36 & W11 & 0.323 & 0.011 \\
\hline HD 39881 & 6.60 & 0.65 & 17 & - & 13.56 & 0.50 & 1 & & & 0.157 & 0.002 \\
\hline HD 41330 & 6.12 & 0.60 & 39 & + & 23.44 & 0.40 & 1 & & & 0.150 & 0.004 \\
\hline HD 42807 & 6.44 & 0.68 & 32 & $-1+$ & 11.77 & 0.08 & 2 & & & 0.364 & 0.011 \\
\hline HD 43162 & 6.37 & 0.68 & 28 & - & 7.077 & 0.022 & 3 & & & 0.419 & 0.021 \\
\hline HD 43587 & 5.70 & 0.59 & 21 & $-1+$ & & & & & & 0.155 & 0.003 \\
\hline HD 45067 & 5.90 & 0.53 & 34 & & & & & 8.0 & $\mathrm{C} 07$ & 0.142 & 0.004 \\
\hline HD 58855 & 5.36 & 0.42 & 43 & $-1+$ & 16.27 & 0.21 & 1 & & & 0.151 & 0.004 \\
\hline HD 61421 & 0.37 & 0.42 & 45 & $-1+$ & & & & & & 0.164 & 0.002 \\
\hline HD 72905 & 5.64 & 0.62 & 49 & & 5.227 & 0.015 & 2 & 4.69 & D96 & 0.400 & 0.009 \\
\hline HD 72945 & 5.92 & 0.51 & 23 & $+/-$ & & & & & & 0.146 & 0.003 \\
\hline HD 75332 & 6.22 & 0.50 & 41 & + & 3.870 & 0.009 & 1 & 4 & B96 & 0.281 & 0.010 \\
\hline HD 75528 & 6.38 & 0.63 & 34 & $-1+$ & & & & & & 0.142 & 0.005 \\
\hline HD 75732 & 5.95 & 0.87 & 34 & & 40.7 & 0.7 & 3 & 42.0 & B98 & 0.177 & 0.008 \\
\hline HD 79028 & 5.20 & 0.51 & 39 & - & 30.8 & 0.7 & 1 & & & 0.146 & 0.004 \\
\hline HD 84117 & 4.94 & 0.53 & 31 & $-/+$ & 5.970 & 0.025 & 1 & & & 0.150 & 0.004 \\
\hline HD 84737 & 5.10 & 0.62 & 31 & $+/-$ & 4.296 & 0.017 & 2 & & & 0.142 & 0.004 \\
\hline HD 86728 & 5.40 & 0.62 & 33 & $+/-$ & 10.64 & 0.14 & 1 & & & 0.150 & 0.005 \\
\hline HD 89744 & 5.74 & 0.49 & 40 & & & & & 12.3 & N84 & 0.140 & 0.006 \\
\hline HD 95128 & 5.04 & 0.62 & 38 & - & 24.8 & 0.8 & 1 & 22.7 & S10 & 0.152 & 0.005 \\
\hline HD 97334 & 6.41 & 0.61 & 28 & $+/-$ & 7.93 & 0.05 & 2 & 7.60 & W11 & 0.356 & 0.012 \\
\hline
\end{tabular}

Notes. Sig: 1: low significance (between 1 and $2 \sigma$ ), 2: between 2 and $3 \sigma ; 3: 3 \sigma$ and higher. For question marks in Col. 6 see Sect. 4 . Asterisks in Col. 11 mark preliminary estimates because Eq. (1) needs to be improved with more highly active stars in future. References in Col. 10 : W11 Wright et al. (2011), N84 Noyes et al. (1984), C07 Cincunegui et al. (2007), H00 Henry et al. (2000), B96 Baliunas et al. (1996), B98 Baliunas et al. (1998), S80 Stimets \& Giles (1980), D93 Donahue (1993), SF87 Simon \& Fekel (1987), S10 Simpson et al. (2010). 
Table A.1. continued.

\begin{tabular}{|c|c|c|c|c|c|c|c|c|c|c|c|}
\hline Star & $V$ & $B-V$ & $N$ & Trend & Period [d] & $\Delta \mathrm{P}[\mathrm{d}]$ & Sig & $P_{\text {lit }}$ & Ref. & $\left\langle S_{\mathrm{MWO}}\right\rangle$ & $\sigma$ \\
\hline HD 97344 & 6.43 & 0.93 & 15 & & & & & & & 0.129 & 0.006 \\
\hline HD 100563 & 5.70 & 0.53 & 32 & $+/-$ & 7.73 & 0.04 & 1 & & & 0.198 & 0.006 \\
\hline HD 100623 & 5.98 & 0.81 & 31 & & 27.5 & 0.5 & 1 & & & 0.194 & 0.008 \\
\hline HD 101501 & 5.34 & 0.74 & 34 & & 17.04 & 0.09 & 3 & 16.68 & W11 & 0.334 & 0.002 \\
\hline HD 106116 & 7.50 & 0.65 & 20 & - & 8.671 & 0.099 & 1 & & & 0.156 & 0.005 \\
\hline HD 106516 & 6.11 & 0.46 & 30 & & 6.63 & 0.04 & 1 & 6.91 & W11 & 0.204 & 0.005 \\
\hline HD 111395 & 6.29 & 0.69 & 35 & $+/-$ & 14.05 & 0.17 & 3 & & & 0.261 & 0.016 \\
\hline HD 114378 & 4.85 & 0.49 & 30 & $-1+$ & 4.394 & 0.015 & 1 & 3.00 & N84 & 0.241 & 0.005 \\
\hline HD 115043 & 6.82 & 0.58 & 41 & & 5.861 & 0.015 & 2 & 6.00 & W11 & 0.314 & 0.011 \\
\hline HD 115383 & 5.22 & 0.59 & 30 & & & & & 3.33 & W11 & 0.323 & 0.006 \\
\hline HD 115617 & 4.74 & 0.70 & 37 & & 26.5 & 0.6 & 1 & 29.0 & W11 & 0.163 & 0.005 \\
\hline HD 117176 & 4.97 & 0.71 & 49 & $+/-$ & 23.44 & 0.40 & 1 & 31.0 & $\mathrm{H} 0 \mathrm{O}$ & 0.147 & 0.004 \\
\hline HD 118972 & 6.92 & 0.82 & 27 & - & 9.407 & 0.049 & 3 & & & $0.574 *$ & 0.019 \\
\hline HD 120136 & 4.49 & 0.49 & 84 & & $114 ?$ & 5 & 3 & 3.20 & $\mathrm{H} 0 \mathrm{O}$ & 0.193 & 0.005 \\
\hline HD 121370 & 2.68 & 0.57 & 36 & $+/-$ & $2.946,8.44$ & $0.008,0.07$ & 2,1 & & & 0.144 & 0.005 \\
\hline HD 124570 & 5.50 & 0.58 & 32 & - & & & & 26 & B96 & 0.134 & 0.005 \\
\hline HD 124850 & 4.08 & 0.52 & 34 & & & & & 7.60 & N84 & 0.216 & 0.006 \\
\hline HD 126053 & 6.27 & 0.63 & 47 & - & 26.28 & 0.53 & 1 & & & 0.172 & 0.005 \\
\hline HD 129333 & 7.61 & 0.59 & 54 & & 2.620 & 0.005 & 2 & 2.67 & W11 & $0.630 *$ & 0.017 \\
\hline HD 131156 & 4.59 & 0.78 & 25 & & 6.299 & 0.037 & 1 & 6.31 & W11 & 0.473 & 0.010 \\
\hline HD 131977 & 5.72 & 1.11 & 30 & $+/-$ & 33.1 & 0.5 & 3 & & & 0.465 & 0.026 \\
\hline HD 133640 & 4.76 & 0.65 & 49 & $+/-$ & & & & & & 0.275 & 0.052 \\
\hline HD 137107 & 5.58 & 0.54 & 30 & & 22.1 & 0.3 & 1 & & & 0.184 & 0.004 \\
\hline HD 140538 & 5.88 & 0.65 & 33 & & & & & & & 0.239 & 0.007 \\
\hline HD 142373 & 4.62 & 0.57 & 37 & - & & & & & & 0.148 & 0.005 \\
\hline HD 145825 & 6.54 & 0.67 & 11 & & & & & & & 0.200 & 0.010 \\
\hline HD 146233 & 5.50 & 0.65 & 34 & $+/-$ & & & & & & 0.170 & 0.004 \\
\hline HD 150706 & 7.03 & 0.57 & 24 & & & & & & & 0.294 & 0.013 \\
\hline HD 157214 & 5.39 & 0.62 & 30 & & & & & & & 0.156 & 0.001 \\
\hline HD 158614 & 5.31 & 0.72 & 13 & & 5.00 & 0.01 & 1 & & & 0.157 & 0.005 \\
\hline HD 159222 & 6.56 & 0.65 & 25 & & 17.42 & 0.22 & 1 & & & 0.182 & 0.006 \\
\hline HD 159332 & 5.66 & 0.45 & 14 & & 25.6 & 0.3 & 1 & & & 0.137 & 0.003 \\
\hline HD 168009 & 6.31 & 0.60 & 19 & $-/+$ & 5.985 & 0.019 & 2 & & & 0.158 & 0.004 \\
\hline HD 178428 & 6.07 & 0.70 & 8 & + & & & & 22.0 & W11 & 0.152 & 0.003 \\
\hline HD 186408 & 5.95 & 0.64 & 8 & & & & & & & 0.150 & 0.003 \\
\hline HD 186427 & 6.20 & 0.66 & 5 & & & & & 31.0 & $\mathrm{H} 0 \mathrm{O}$ & 0.153 & 0.006 \\
\hline HD 190771 & 6.17 & 0.65 & 8 & & & & & & & 0.357 & 0.009 \\
\hline HD 196850 & 6.78 & 0.59 & 10 & & & & & & & 0.155 & 0.002 \\
\hline HD 197076 & 6.44 & 0.62 & 6 & & & & & & & 0.175 & 0.004 \\
\hline HD 201091 & 5.21 & 1.18 & 16 & + & 34.1 & 0.3 & 2 & 35.37 & W11 & $0.567 *$ & 0.025 \\
\hline HD 207978 & 5.53 & 0.42 & 18 & + & 3.197 & 0.003 & 1 & 3 & B96 & 0.146 & 0.003 \\
\hline HD 216385 & 5.16 & 0.48 & 16 & + & & & & & & 0.138 & 0.003 \\
\hline HD 217014 & 5.46 & 0.70 & 13 & + & 38.0 & 0.6 & 1 & 37.0 & W11 & 0.150 & 0.003 \\
\hline HD 224930 & 5.75 & 0.67 & 14 & & 15.01 & 0.05 & 2 & 33 & B96 & 0.181 & 0.004 \\
\hline
\end{tabular}




\section{Appendix B: Plots}
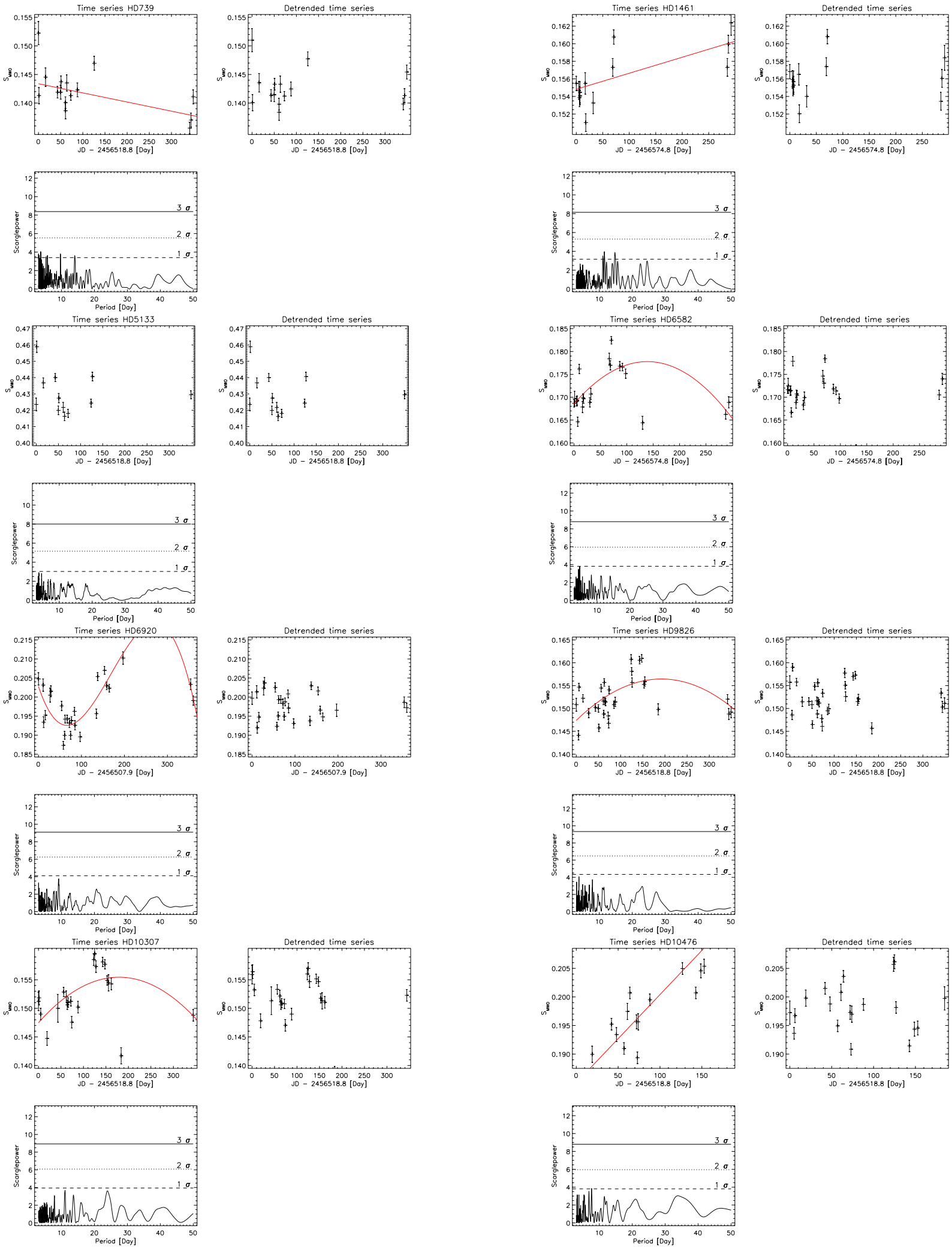

Fig. B.1. Plots of individual stars. Each figure contains the original time series (top left) with HD number, including a polynomial fit (full line) to eliminate a long-term trend, the time series corrected for a long-term trend (top right), the Scargle periodogram (bottom left) and a phase plot (bottom right). 
A. Hempelmann et al.: Measuring rotation periods of solar-like stars using TIGRE
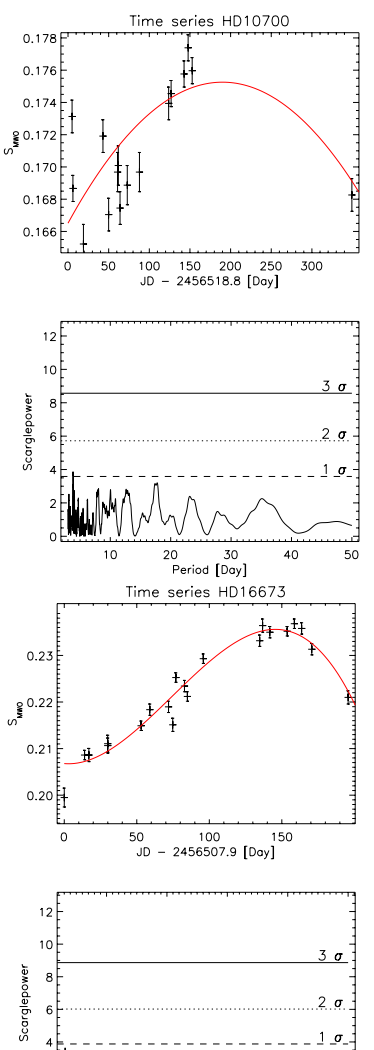

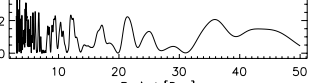
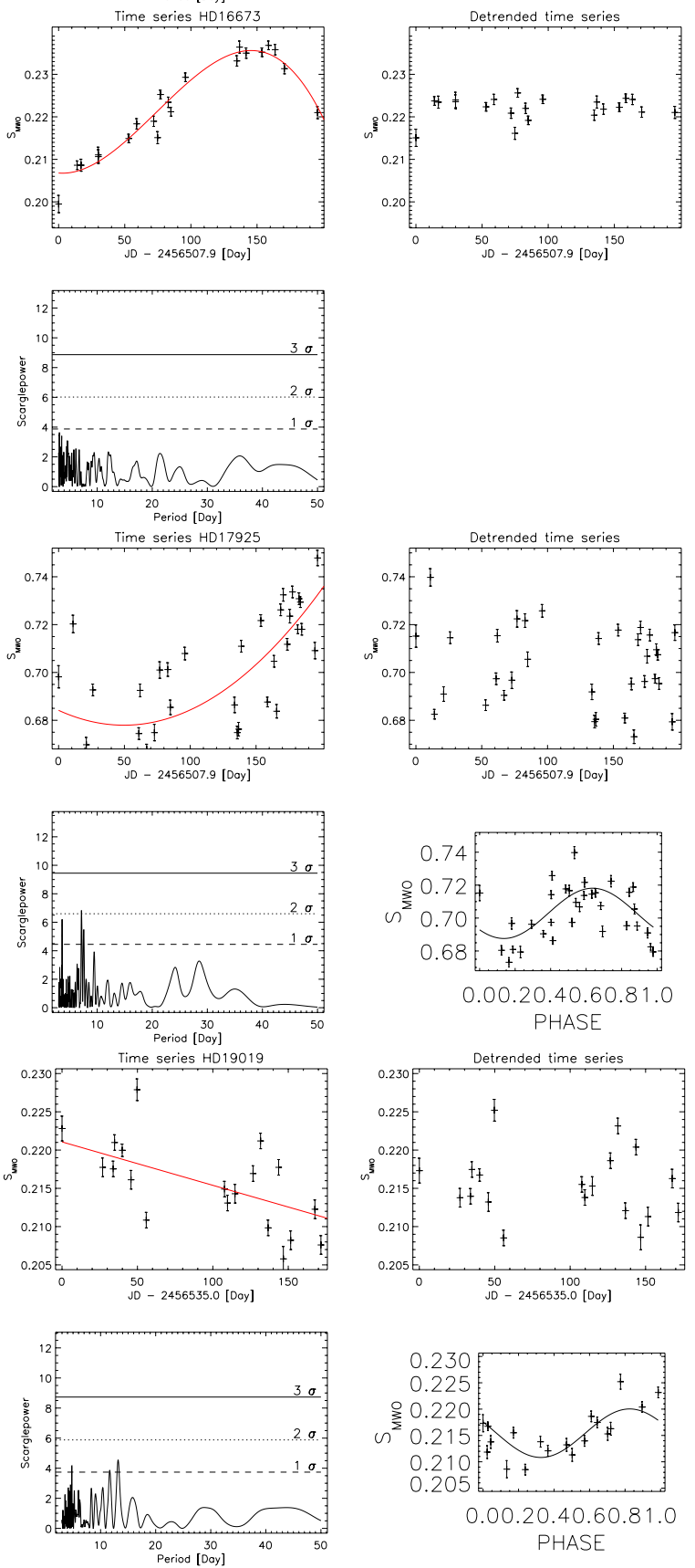
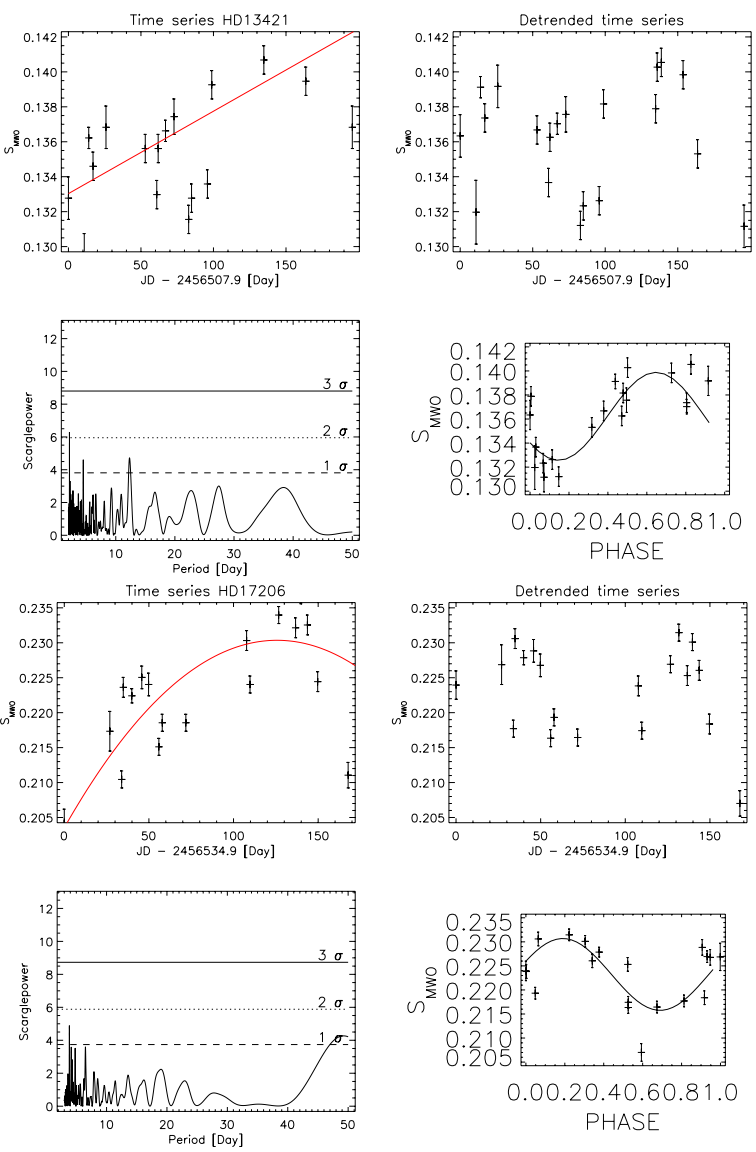

0.00 .20 .40 .60 .81 .0 PHASE
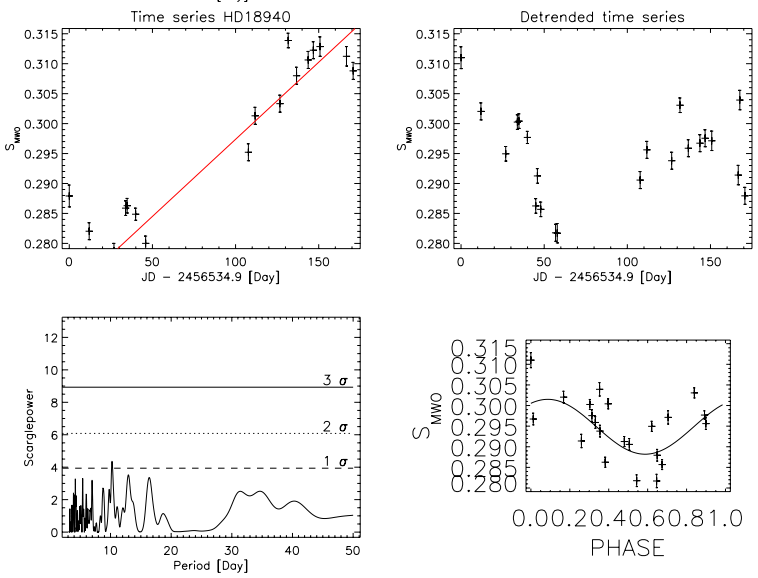

0.00 .20 .40 .60 .81 .0 PHASE
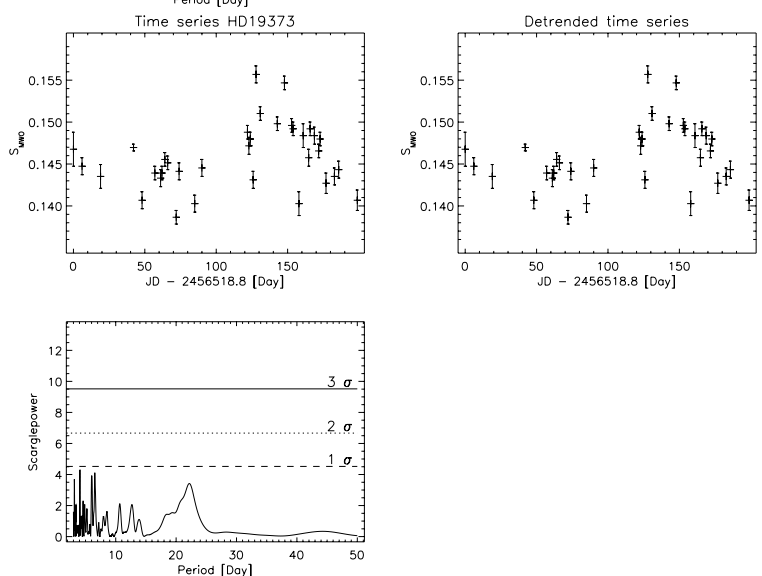

Fig. B.1. continued. 
A\&A 586, A14 (2016)
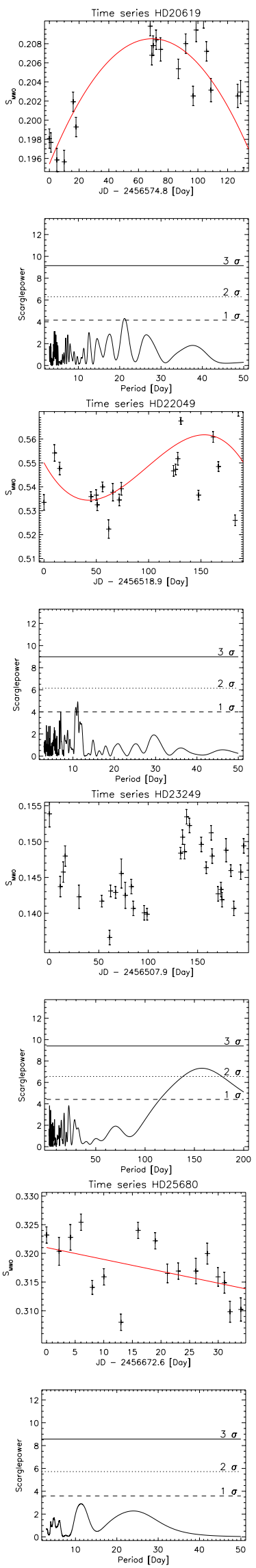

Fig. B.1. continued.
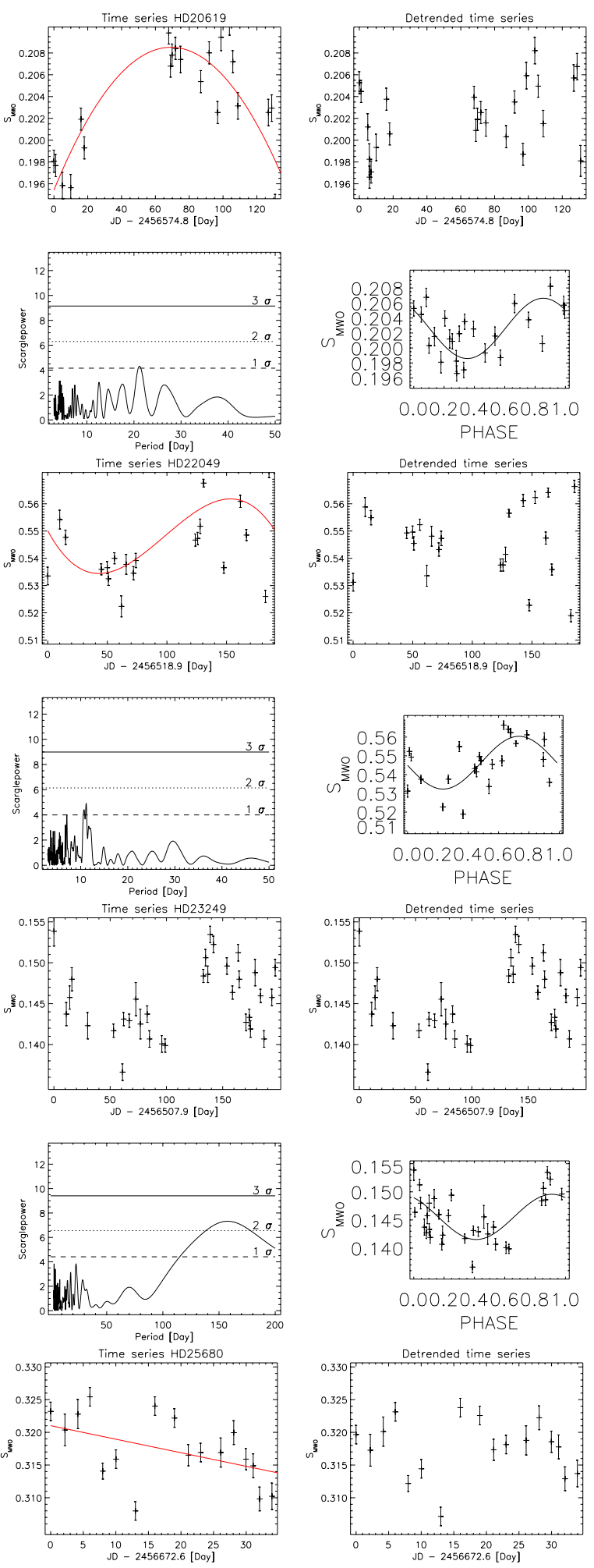

A14, page 10 of 19
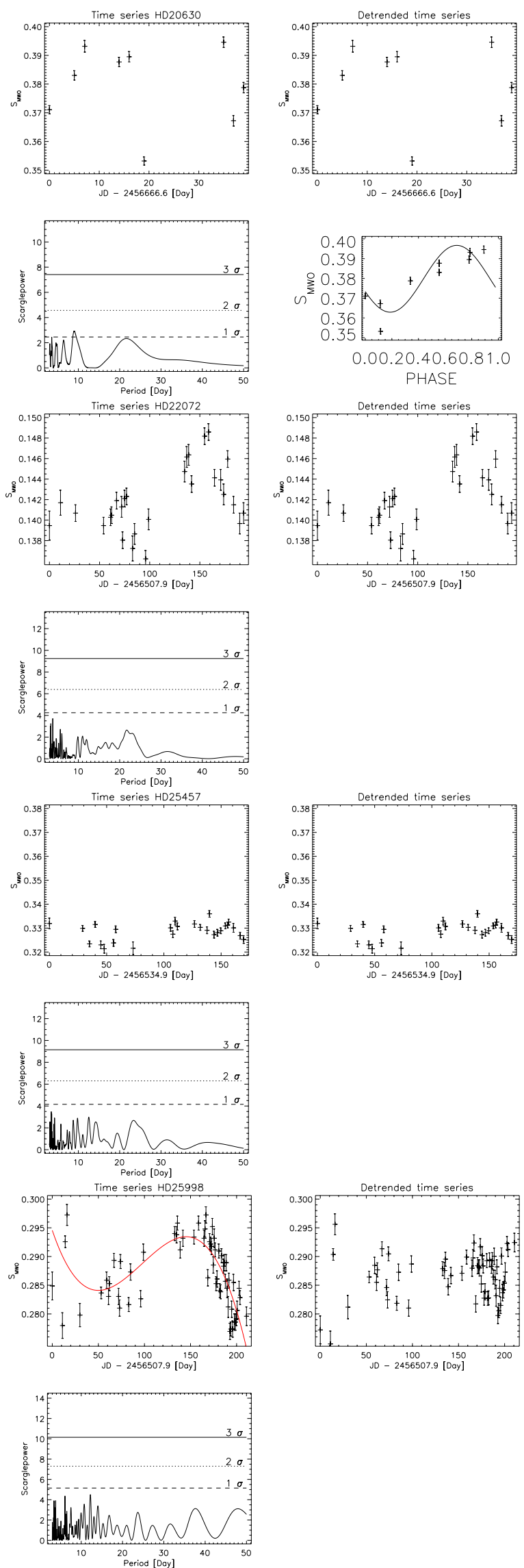
A. Hempelmann et al.: Measuring rotation periods of solar-like stars using TIGRE
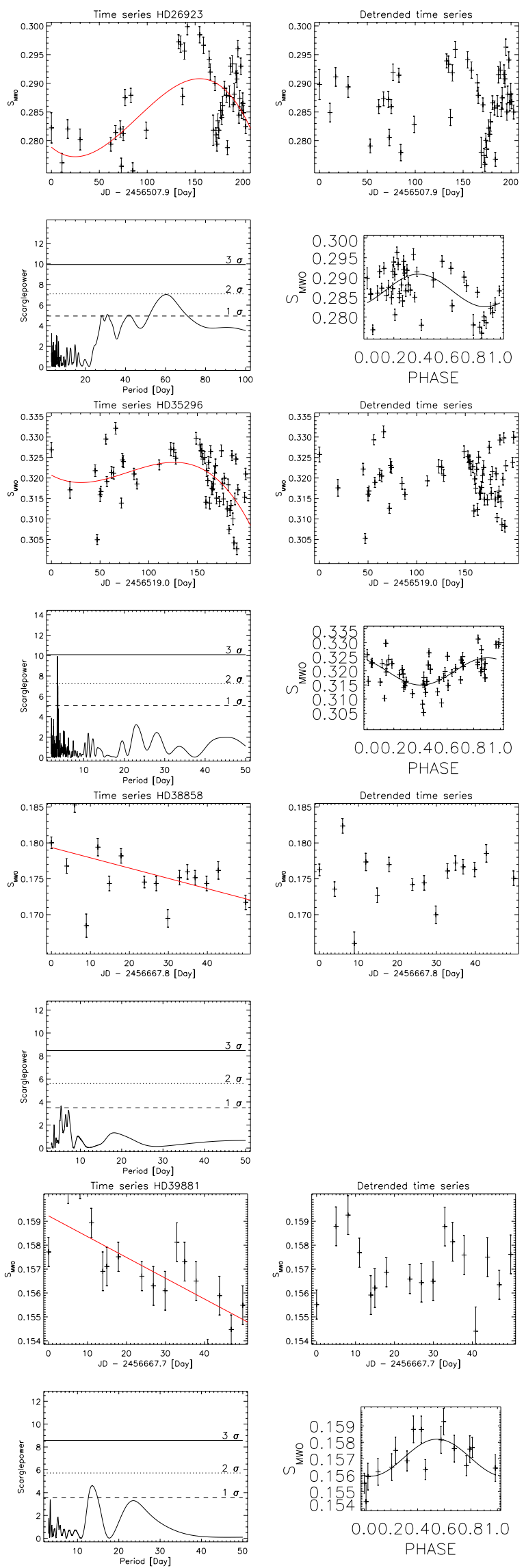

0.00 .20 .40 .60 .81 .0 PHASE
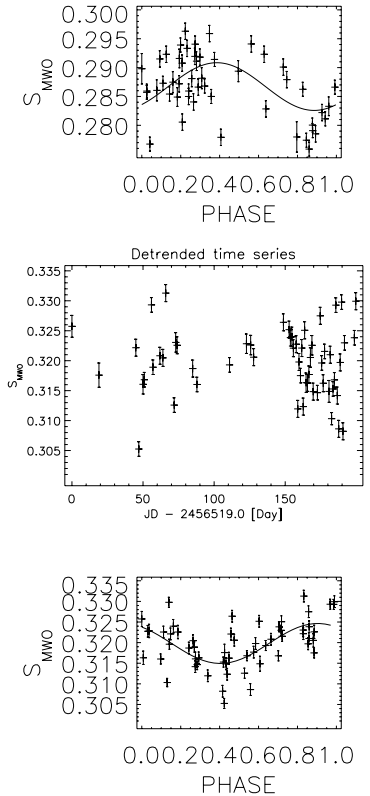
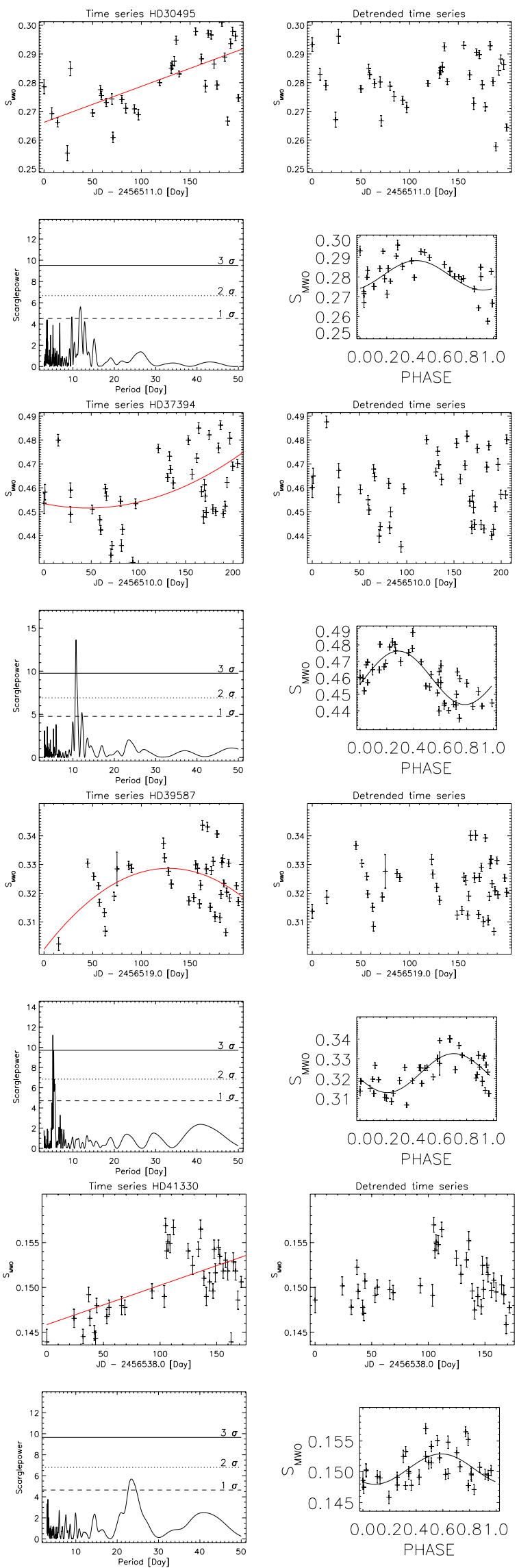

Fig. B.1. continued. 
A\&A 586, A14 (2016)
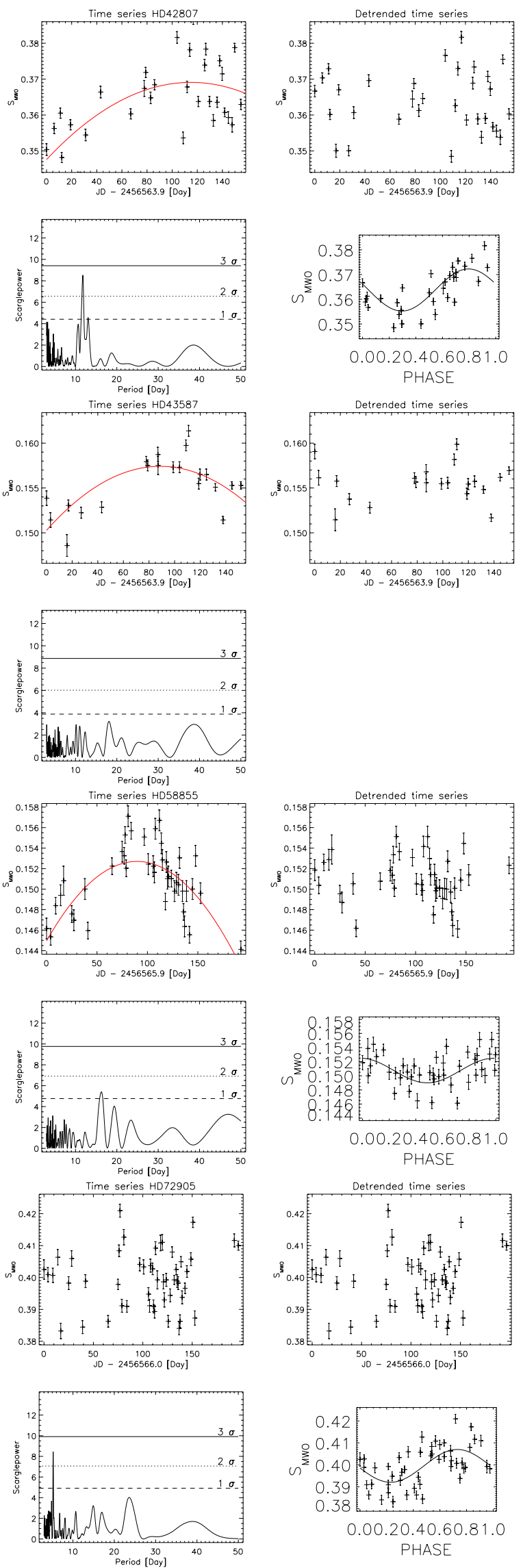
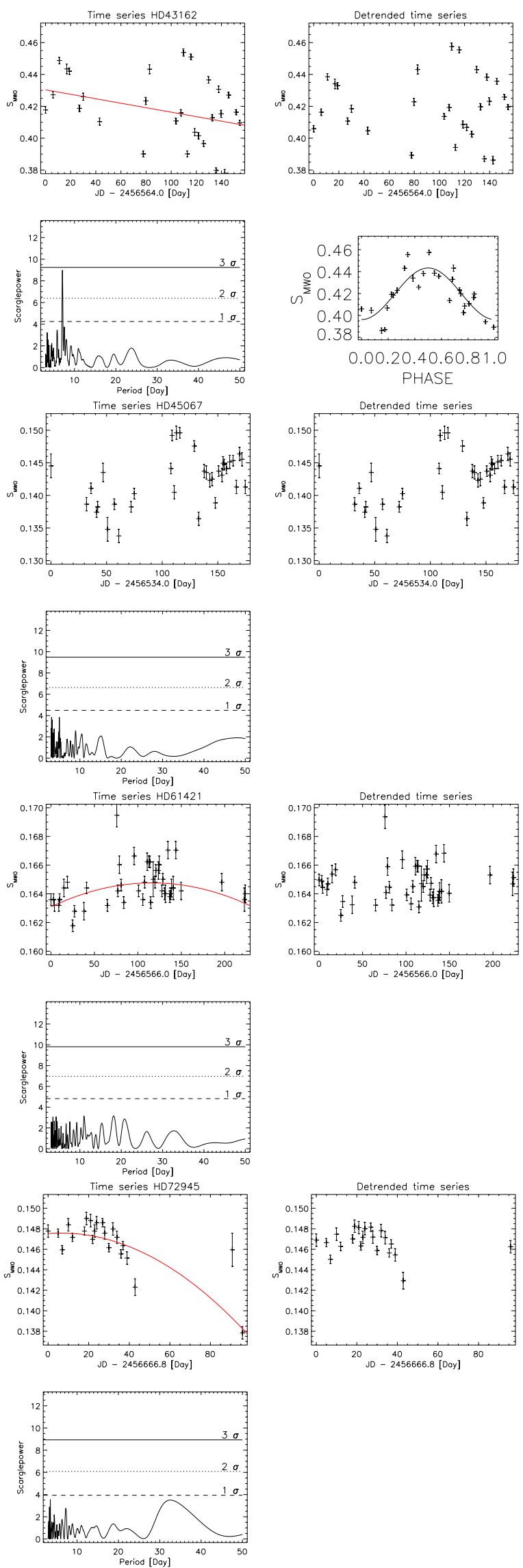

Fig. B.1. continued. 
A. Hempelmann et al.: Measuring rotation periods of solar-like stars using TIGRE
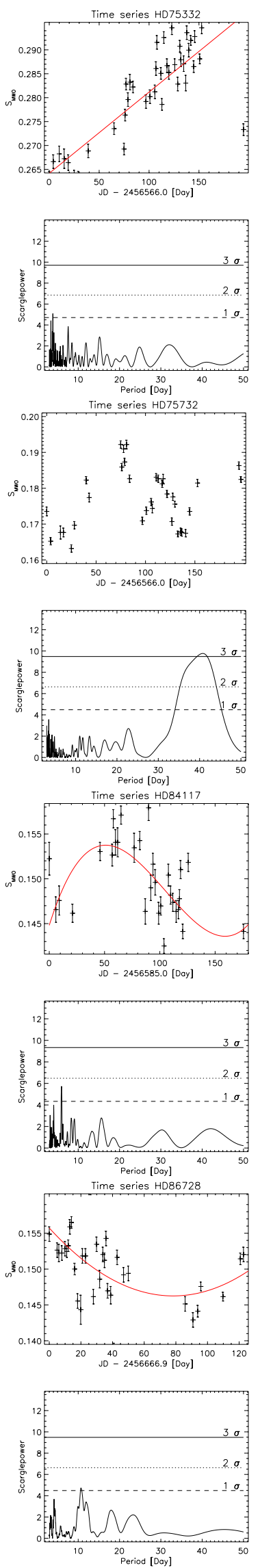

Fig. B.1. continued.
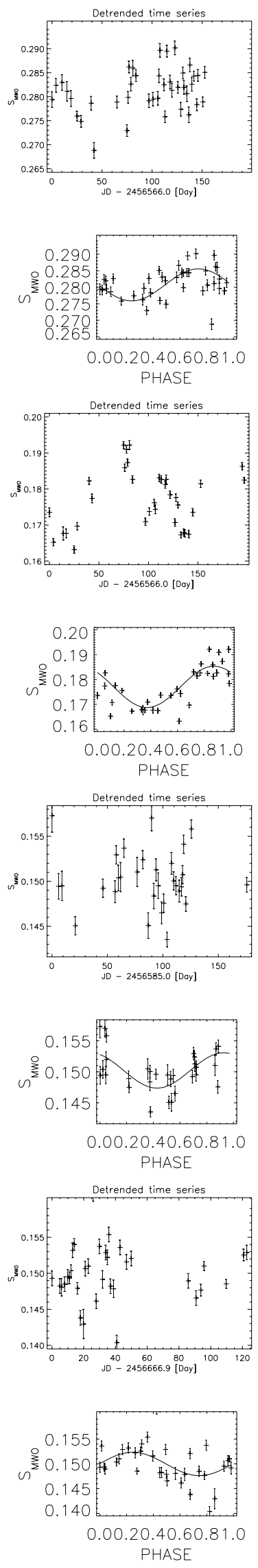
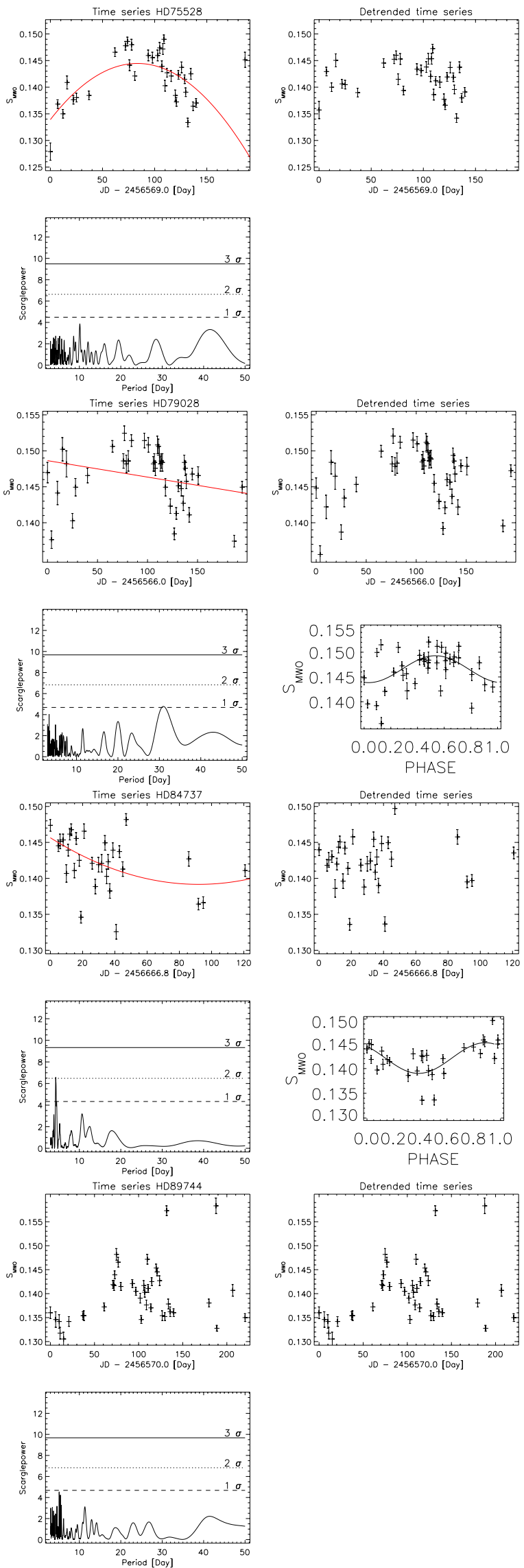
A\&A 586, A14 (2016)
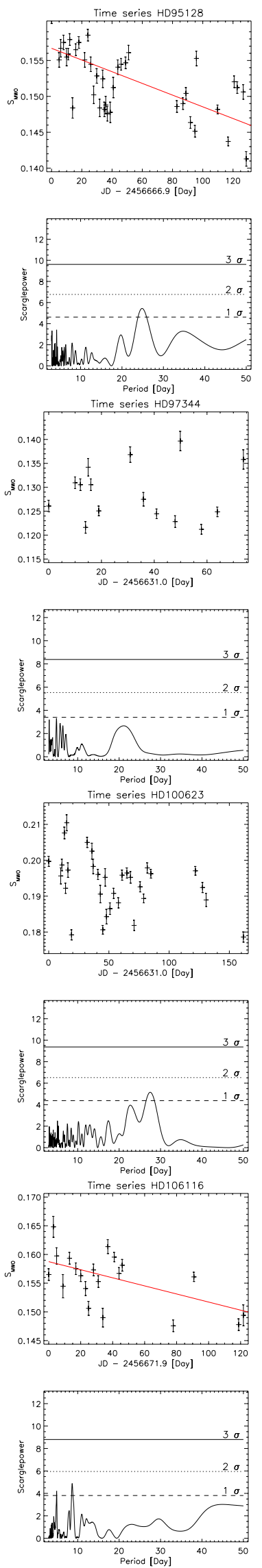

Fig. B.1. continued.
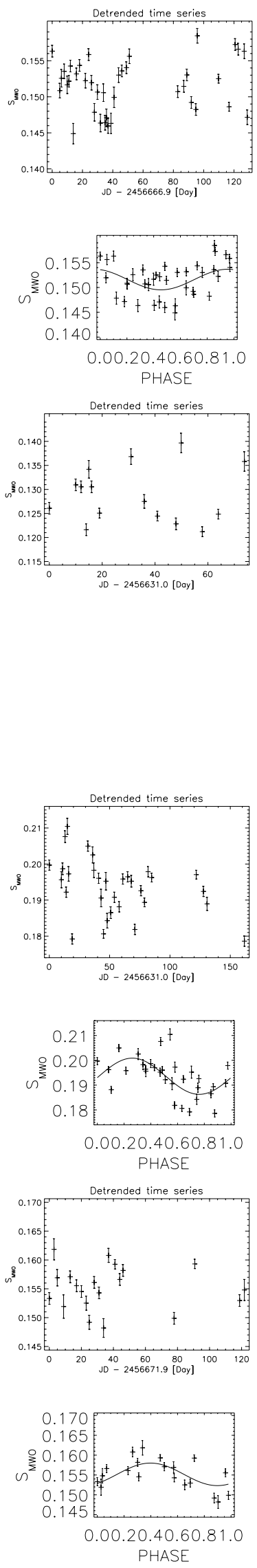
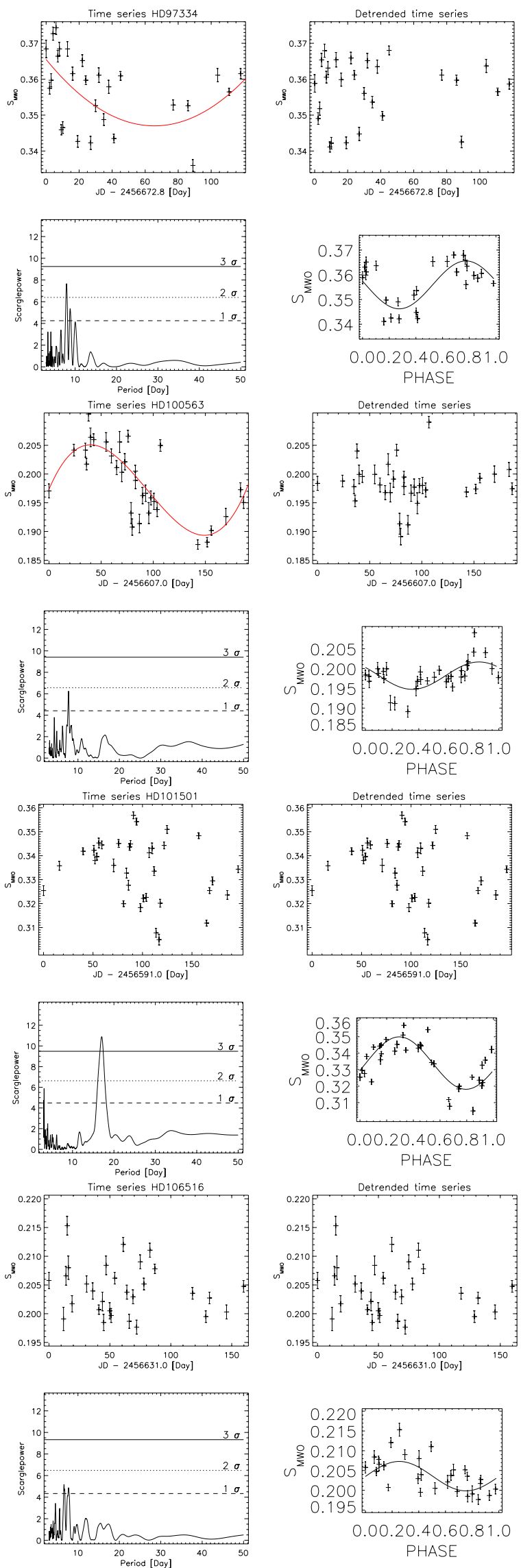
A. Hempelmann et al.: Measuring rotation periods of solar-like stars using TIGRE
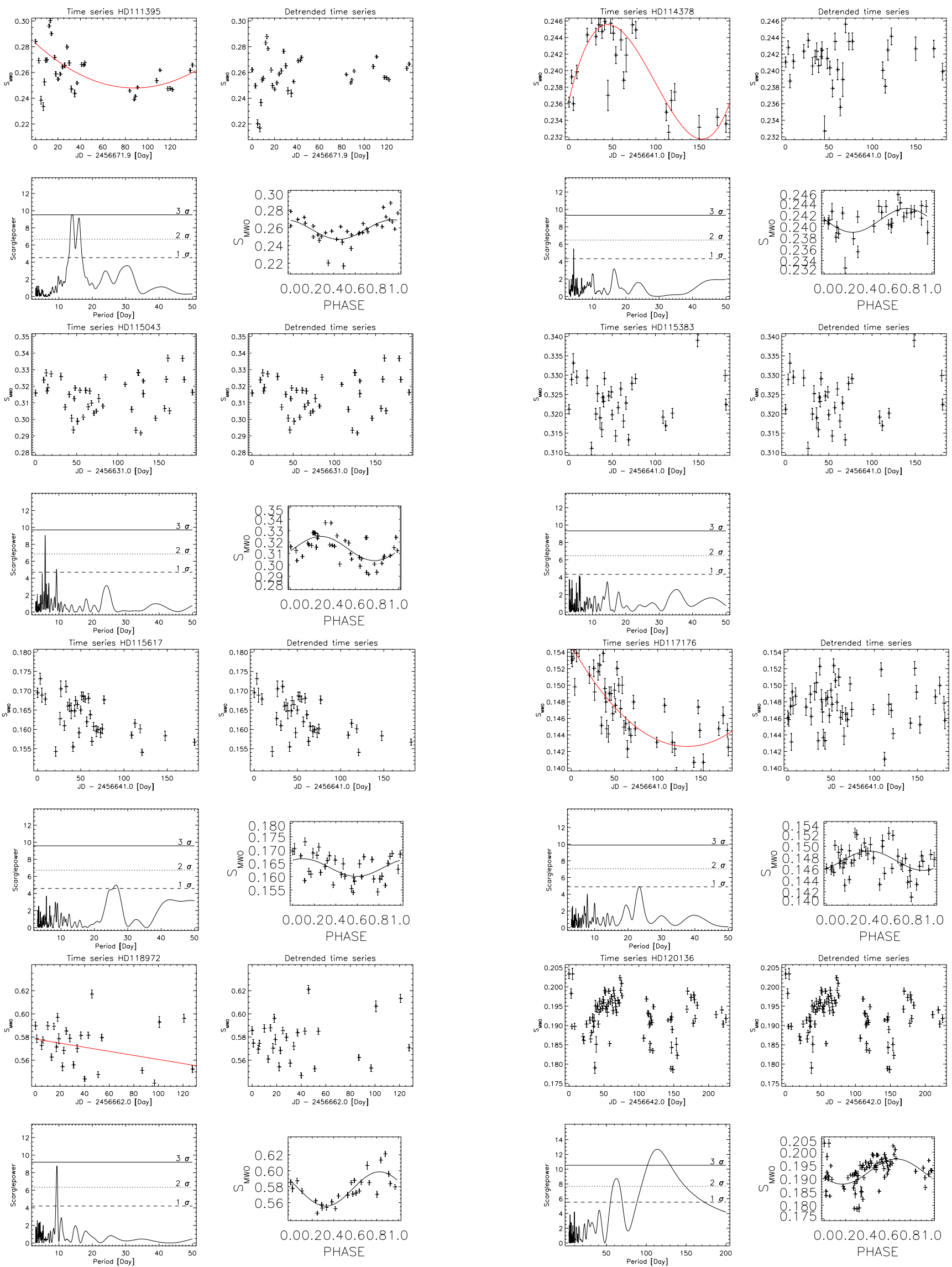

Fig. B.1. continued. 
A\&A 586, A14 (2016)
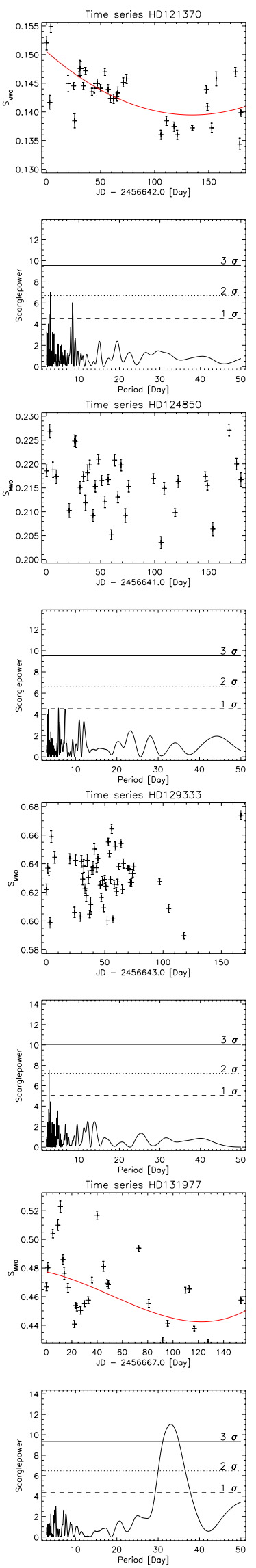

Fig. B.1. continued.
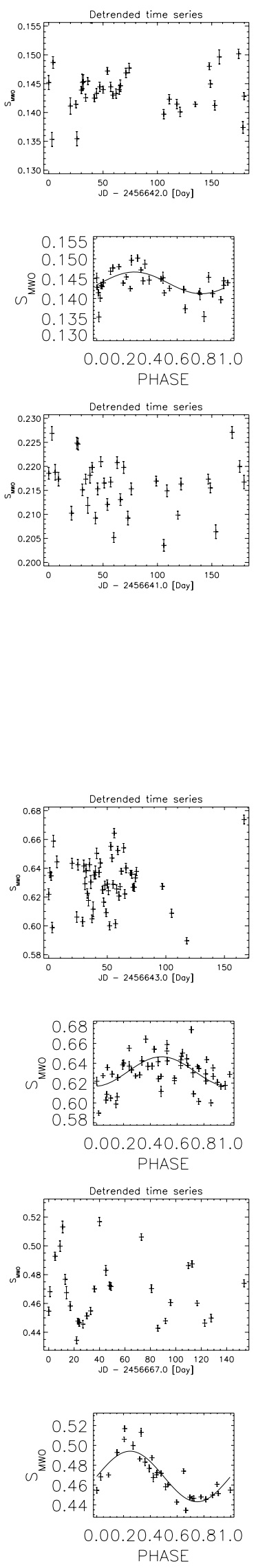
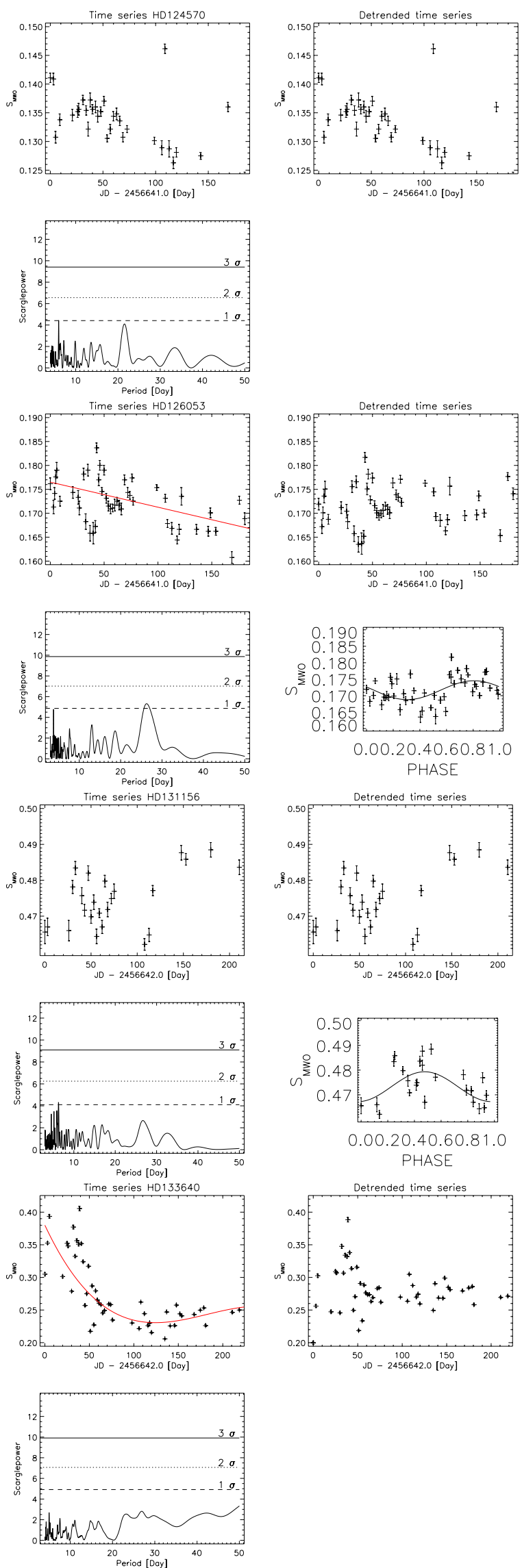
A. Hempelmann et al.: Measuring rotation periods of solar-like stars using TIGRE
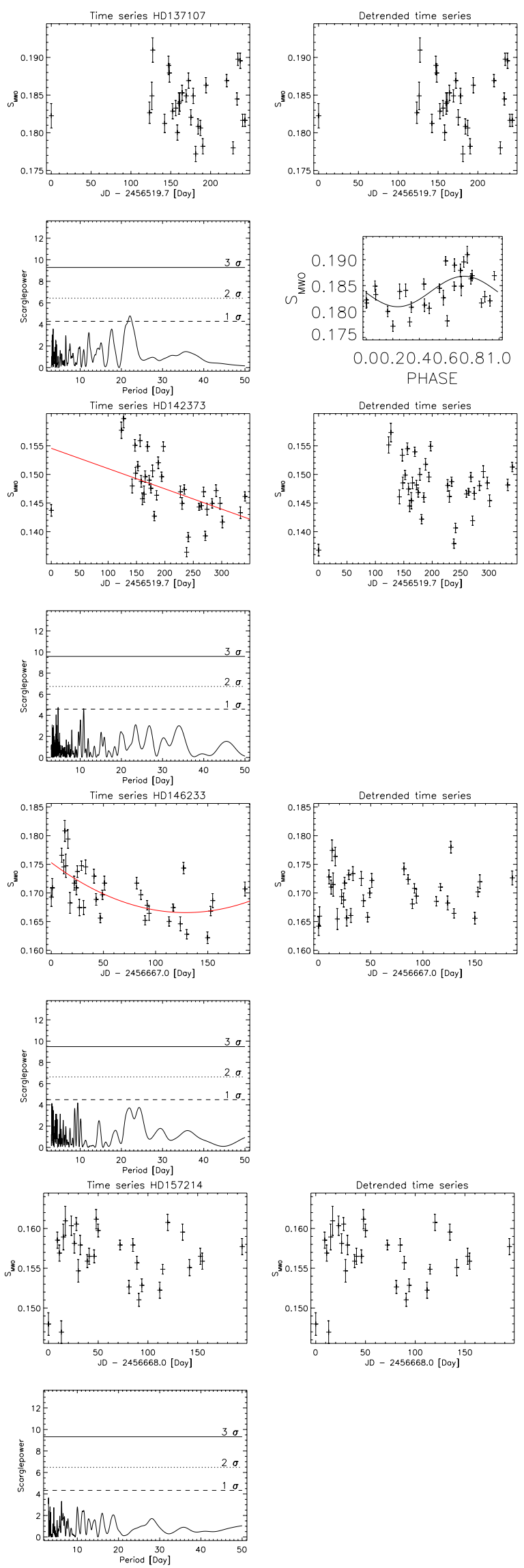

Fig. B.1. continued.
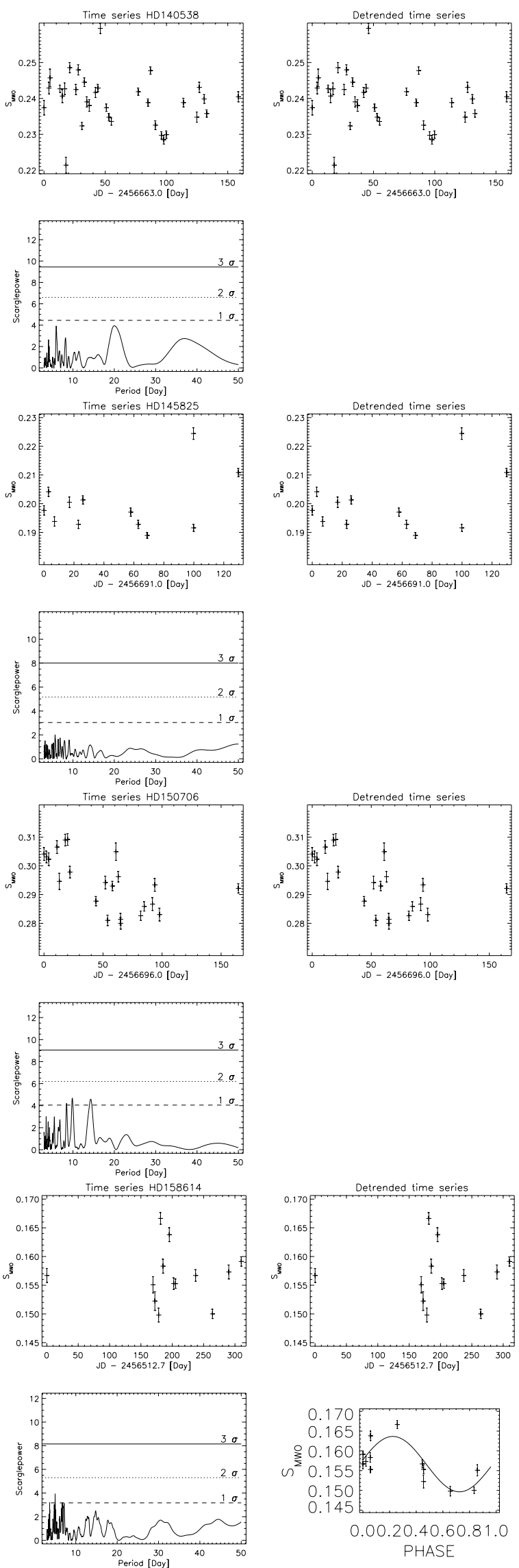
A\&A 586, A14 (2016)
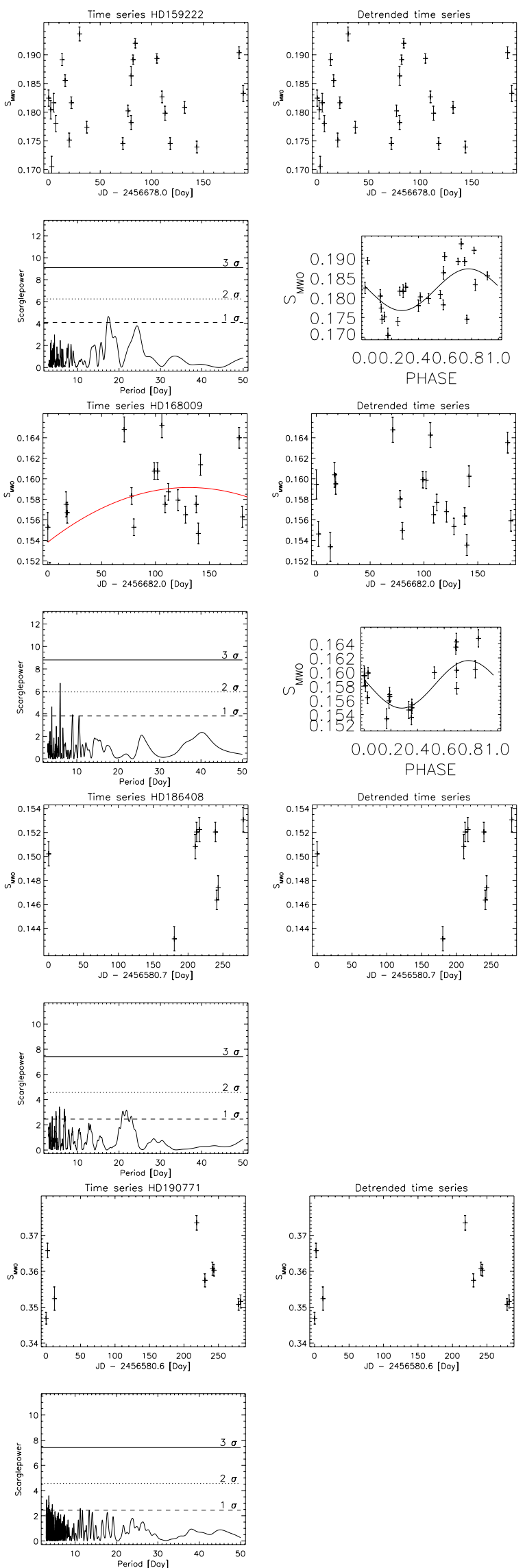

Fig. B.1. continued.
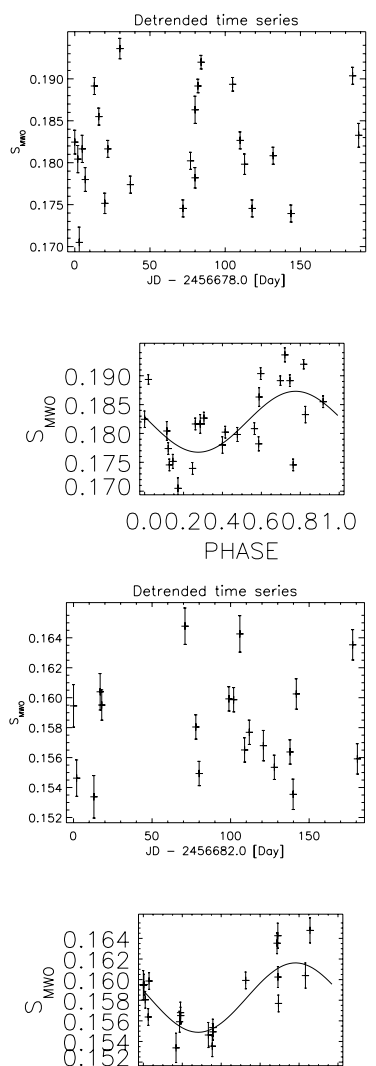

0.00 .20 .40 .60 .81 .0 PHASE

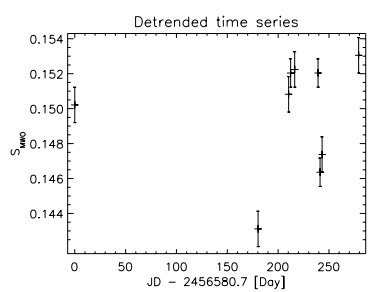

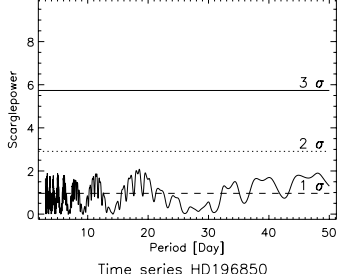
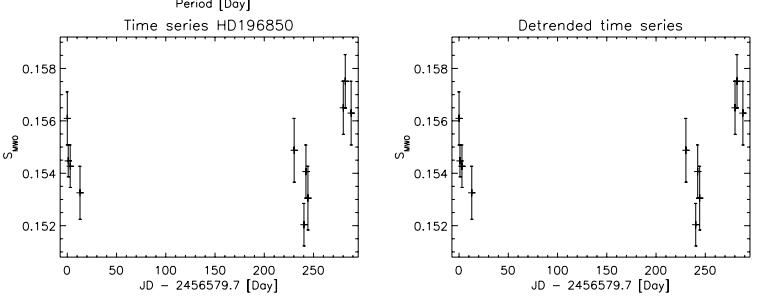
A. Hempelmann et al.: Measuring rotation periods of solar-like stars using TIGRE
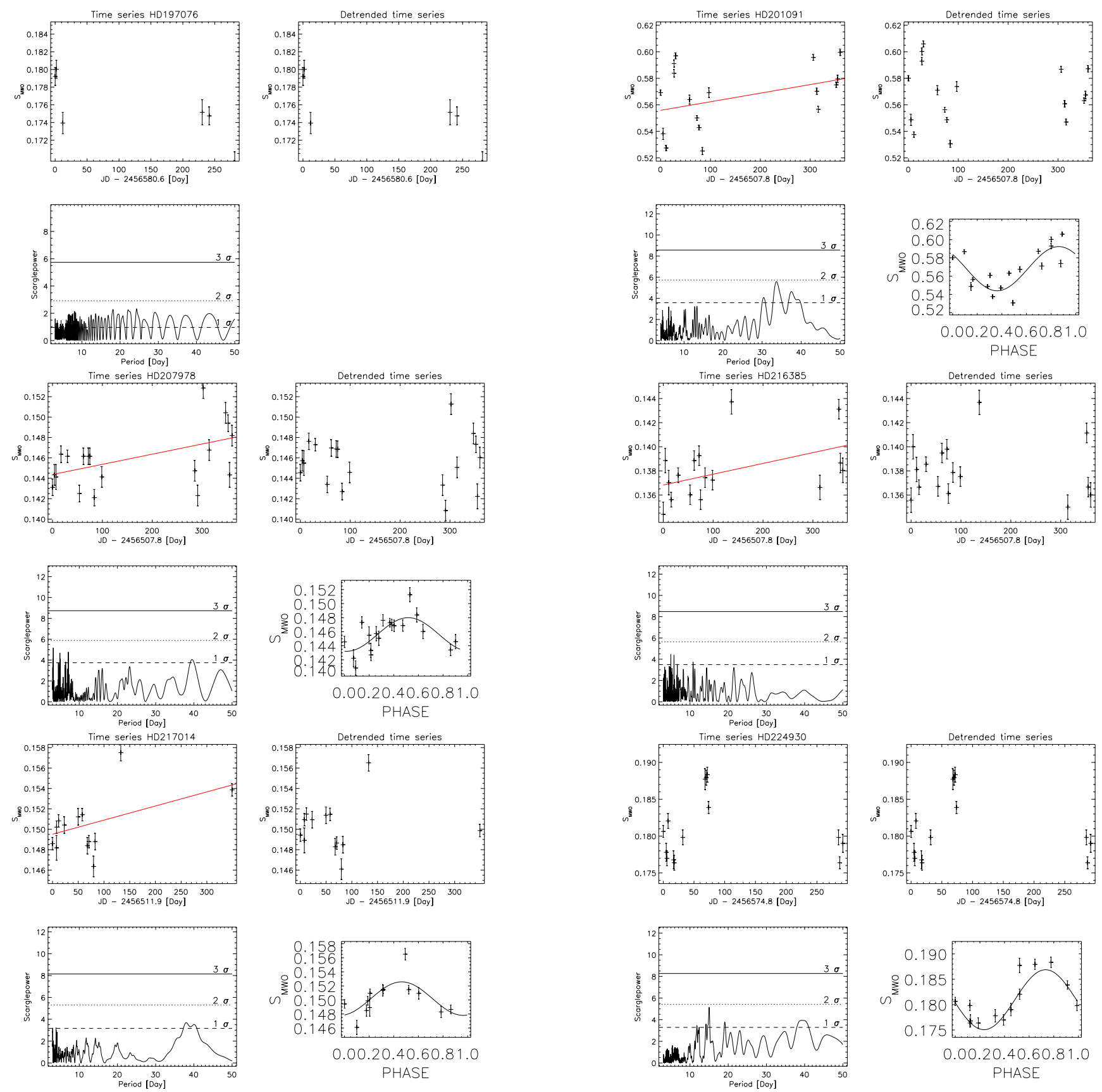

Fig. B.1. continued. 